\title{
HMGB1 participates in LPS-induced acute lung injury by activating the AIM2 inflammasome in macrophages and inducing polarization of M1 macrophages via TLR2, TLR4, and RAGE/NF-кB signaling pathways
}

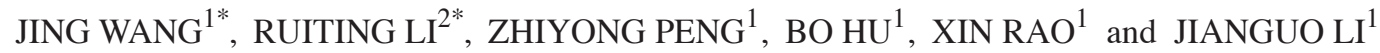 \\ ${ }^{1}$ Department of Intensive Care Unit, Zhongnan Hospital of Wuhan University, Wuhan, Hubei 430071; \\ ${ }^{2}$ Department of Critical Care Unit, Institute of Anesthesia and Critical Care Medicine, Union Hospital, \\ Tongji Medical College, Huazhong University of Science and Technology, Wuhan, Hubei 430022, P.R. China
}

Received May 5, 2019; Accepted September 30, 2019

DOI: 10.3892/ijmm.2019.4402

\begin{abstract}
High mobility group box 1 (HMGB1), a crucial proinflammatory cytokine, was reported to activate the absent in melanoma 2 (AIM2) inflammasome, which are both essential in acute lung injury (ALI). However, their interaction mechanism has remained elusive. Macrophages are known to express the AIM2 inflammasome and the main receptors [receptor for advanced glycation end products (RAGE), Toll-like receptor 2/4 (TLR-2/TLR-4)] of HMGB1 to transmit intracellular signals. The present study aimed to indicate whether HMGB1 participates in the process of lipopolysaccharides (LPS)-induced ALI through activating the AIM2 inflammasome in macrophages, as well as inducing polarization of M1 macrophages via TLR2, TLR4 and RAGE/ nuclear factor $-\kappa \mathrm{B}(\mathrm{NF}-\kappa \mathrm{B})$ signaling pathways. In an in vivo mouse model of LPS-induced ALI, anti-HMGB1, recombinant (r) HMGB1, LPS from Rhodobacter sphaeroides (LPS-RS, TLR2/4 antagonist) or FPS-ZM1 (RAGE antagonist) were administrated. In in vitro studies, bone marrow-derived macrophages from mice primed with LPS were stimulated with or without anti-HMGB1, rHMGB1, LPS-RS, or FPS-ZM1. The findings revealed that anti-HMGB1, LPS-RS and FPS-ZM1 significantly decreased infiltration of inflam-
\end{abstract}

Correspondence to: Professor Jianguo Li, Department of Intensive Care Unit, Zhongnan Hospital of Wuhan University, 169 Donghu Road, Wuchang, Wuhan, Hubei 430071, P.R. China

E-mail: drljg1819@163.com

*Contributed equally

Abbreviations: HMGB1, high mobility group box 1; AIM2, absent in melanoma 2; ALI, acute lung injury; NF- $\kappa \mathrm{B}$ nuclear factor- $\kappa \mathrm{B}$; W/D, wet-to-dry; MPO, myeloperoxidase; BALF, bronchoalveolar lavage fluid; DCs, dendritic cells; H\&E, hematoxylin and eosin

Key words: acute lung injury, high mobility group box 1, polarization of M1 macrophages, absent in melanoma 2 inflammasome, cytokines matory cells, wet-to-dry ratio, myeloperoxidase activity in the lung, the levels of cytokines, as well as macrophages and neutrophil infiltration in the bronchoalveolar lavage fluid. However, rHMGB1 aggravated the inflammatory response in ALI. Mechanistically, anti-HMGB1, LPS-RS and FPS-ZM1 attenuated activation of TLR2, TLR4, and RAGE/NF- $\kappa$ B signaling pathways and expression of the AIM2 inflammasome in macrophages. However, rHMGB1 enhanced their expression levels and induced polarization of M1 macrophages. These results indicated that HMGB1 could participate in the pathogenesis of ALI by activating the AIM2 inflammasome in macrophages, as well as inducing polarization of M1 macrophages through TLR2, TLR4 and RAGE/NF- $\kappa$ B signaling pathways.

\section{Introduction}

Acute lung injury (ALI) is characterized by dyspnea and hypoxemia as clinical features, and pulmonary interstitial edema, alveolar epithelial injury, and the acute excessive inflammatory response process are triggered by various pathologies, such as infection, trauma, and shock (1). ALI is accompanied by a high morbidity and mortality rate, and no specific treatment exists (2). As a result, how to target the biomarkers of ALI to inhibit the inflammatory response and the molecular and cellular events during inflammation, to activate the host immune response or produce a balanced relationship between immune and inflammatory responses have attracted scholars' attention $(3,4)$.

High mobility group box 1 protein (HMGB1), a late inflammatory cytokine, is a highly conserved nuclear DNA-binding protein that activates macrophages and dendritic cells (DCs) to produce inflammatory cytokines, mediating systemic inflammatory responses (5-8). Several studies have reported that HMGB1 plays a substantial role in the pathogenesis of ALI and sepsis $(5,6,9)$. The serum and tissue levels of HMGB1 are elevated during ALI and sepsis, and HMGB1 inhibitors can alleviate the inflammatory response, tissue injury, and organ dysfunction $(9,10)$. However, to the best of our knowledge no 
data has been reported about the exact mechanism of action of HMGB1 in the pathogenesis of ALI.

Macrophages, the main proinflammatory cells, can differentiate into classically activated macrophages (M1) and alternatively activated macrophages (M2) (11). Under physiological conditions, there is a balance between M1 and M2 macrophages. M1 macrophages produce interleukin-1 $\beta$ (IL-1 $\beta)$, tumor necrosis factor- $\alpha$ (TNF- $\alpha$ ) and IL-6, which stimulate and activate neutrophils, leading to the release of proteases and oxidant-induced lung damage (12). M2 macrophages can accelerate resolution and lung repair in the acute respiratory distress syndrome (13). In ALI, polarization of macrophages into the M1 phenotype by adapting to the modified microenvironment results in the production of a great number of inflammatory factors (14). Activated macrophages not only secrete HMGB1 into the extracellular environment, but also express receptors at their surface to which HMGB1 binds (8). Receptor for advanced glycation end products (RAGE), Toll-like receptor 2 (TLR2) and TLR4 have been reported as the main receptors of HMGB1 that transmit intracellular signals, leading to activation of the nuclear factor- $\kappa \mathrm{B}$ $(\mathrm{NF}-\kappa \mathrm{B})$ pathway $(5,7,8)$. Therefore, it is possible that HMGB1 activates macrophages via TLR2, TLR4, and RAGE/NF- $\kappa \mathrm{B}$ signaling pathways, and induces polarization of macrophage M1 in ALI.

The absent in melanoma 2 (AIM2) inflammasome, comprising AIM2, the adaptor apoptosis-associated speck-like protein containing a CARD (ASC) and caspase-1, is a major intracellular polyprotein complex of the innate immune system expressed by macrophages, DCs, and other immune cells $(15,16)$. In the development of infection and ALI, AIM2 inflammasomes are activated, leading to the maturation of caspase-1, pro-IL-18 and pro-IL-1 $\beta$, in addition to their release in the serum and tissue, inducing lung inflammation (15-17). In the process of lung inflammation, the expression of AIM2 in macrophages would elevate and the polarization of macrophage M1 polarization would develop. A study also found that M1 polarized macrophages express elevated AIM2 gene expression (18). As a result, in the process of lung injury, macrophages could on one hand activate AIM2 and release inflammatory mediators and lead to inflammatory injury and on the other hand, could develop towards M1 polarization, resulting lung tissue injury. A study revealed that inflammasomes induce the secretion of HMGB1 in the nucleus (19). However, HMGB1 can activate AIM2 inflammasomes to attend to the process of proinflammatory response and innate immunity $(20,21)$. Thus, it was inferred that HMGB1 participates in the process of lipopolysaccharide (LPS)-induced ALI in mice by activating AIM2 inflammasomes in macrophages, as well as inducing polarization of M1 macrophages via TLR2, TLR4 and RAGE/NF- $\kappa$ B signaling pathways.

\section{Materials and methods}

Animals. All animal experiments were approved by the Institutional Animal Care and Use Committee of Wuhan University (Wuhan, China), as well as being in compliance with the Animal Welfare Act. A total of 150 male C57BL/6 mice, aged 6-8 weeks old and weighing 20-22 g, were purchased from the Hubei Experimental Animal Research Center of Hubei province (Wuhan, China) and bred in the Animal Biosafety Level-III Laboratory of Wuhan University. In the laboratory, the mice were kept on a 12 -h light and dark cycle at $25^{\circ} \mathrm{C}$, with a humidity of $45-55 \%$ in a ventilated cage, and with free access to food and water. The animals were assigned to 10 groups (control, LPS, anti-HMGB1, LPS+anti-HMGB1, rHMGB1, LPS+rHMGB1, FPS-ZM1, LPS+FPS-ZM1, LPS+RS and LPS+LPS-RS; n=4-6 per group) and all experiments were repeated more than three times.

LPS-induced model of ALI and experimental treatment. Male mice were anesthetized by intraperitoneal (i.p.) injection of $1 \%$ pentobarbital sodium solution $(80 \mathrm{mg} / \mathrm{kg}$, Biyuntian Institute of Biotechnology) and randomly assigned to the following groups: Control, LPS, positive control, and treatment group ( $n=4-6$ per each group). Mice in the LPS and treatment groups then received an i.p., injection of LPS $(15 \mathrm{mg} / \mathrm{kg}$; Sigma-Aldrich, Merck KGaA) diluted in $200 \mu \mathrm{l}$ sterile PBS. The control and positive control groups were administered only PBS by the same way and volume. The positive control and treatment groups received an intranasal (i.n.) injection of anti-HMGB1 $(2.5 \mu \mathrm{g} / \mathrm{g}$ in $40 \mu \mathrm{l} \mathrm{PBS}, 2 \mathrm{~h}$ after LPS challenge; BioLegend, Inc.) (22) or rHMGB1 $(0.5 \mu \mathrm{g} / \mathrm{g}$ in $40 \mu \mathrm{l} \mathrm{PBS}$, $2 \mathrm{~h}$ after LPS challenge; R\&D Systems, Inc.) (23-25), an i.p., injection of LPS from Rhodobacter sphaeroides (LPS-RS), a TLR2/4 antagonist $(0.1 \mathrm{mg} / \mathrm{mg}$ in $200 \mu$ l endotoxin-free water, $1 \mathrm{~h}$ before LPS challenge; InvivoGen) (25-27) or FPS-ZM1, a RAGE antagonist $(1.5 \mathrm{mg} / \mathrm{kg}$ in $200 \mu \mathrm{l} \mathrm{PBS,} 1 \mathrm{~h}$ before LPS challenge; EMD Millipore) $(25,28)$. All mice were sacrificed (cervical dislocation) $24 \mathrm{~h}$ after the last treatment and the blood, lung, bronchoalveolar lavage fluid (BALF), and spleen of mice were extracted for sample preparation.

Lung wet-to-dry (W/D) ratio. The left lung of the mice was collected and immediately weighed to obtain the 'wet' weight, and then placed in an incubator at $80^{\circ} \mathrm{C}$ for $48 \mathrm{~h}$ to obtain the 'dry' weight. The ratio of W/D was quantified by dividing the lung wet weight by the lung dry weight.

Myeloperoxidase (MPO) activity detection. MPO activity in lung tissue was measured using an MPO assay kit (A044; Nanjing Jiancheng Bioengineering Institute) in accordance with the manufacturer's protocol. Parts of the right lung tissues were homogenized and centrifuged $\left(4^{\circ} \mathrm{C} ; 1,006.2 \mathrm{x} \mathrm{g} ; 30 \mathrm{~min}\right)$. The supernatant was incubated with a substrate buffer (1:10). The enzymatic activity was determined at $450 \mathrm{~nm}$ using a spectrophotometer.

Histological analysis of the lungs. The left lung of mice was removed and fixed in $4 \%$ paraformaldehyde buffer (room temperature; $24 \mathrm{~h}$ ), dehydrated, embedded, and sectioned into $5-\mu \mathrm{m}$ slices, which were stained with hematoxylin and eosin (H\&E) (room temperature; staining with hematoxylin for $5 \mathrm{~min}$, followed by $\mathrm{HCl}$-alcohol differentiation for $20 \mathrm{sec}$, flushing with running water, incubation with $1 \%$ ammonia for $30 \mathrm{sec}$ to return the blue color, further washing in water and staining with eosin for $3 \mathrm{~min}$ ) to evaluate lung inflammation. A light microscope with magnification of $\times 200$ was used to observe the lung tissue. A total of five samples were chosen in each group and five images were acquired for every sample. 
The pathological changes of lung tissues were assessed by the lung injury scores $(29,30)$. Lung injury scores were categorized as follows: Neutrophil infiltration (0-4), interstitial edema (0-4), hemorrhage (0-4) and hyaline membrane formation, necrosis, congestion (0-4). The severity of microscopic injury was judged according to the following scoring system: 0 , Normal; 1, Minimal (<25\%); 2, Mild (25-50\%); 3, Moderate (50-70\%); and 4, Severe (>75\%).

$B A L F$ collection. BALF was collected from mice sacrificed $24 \mathrm{~h}$ after LPS administration to analyze the cellular components and cytokine production in BALF. The lungs were lavaged three times in $0.5 \mathrm{ml}$ PBS and BALF was centrifuged at $503.1 \mathrm{x} \mathrm{g}$ for $7 \mathrm{~min}$ at $4^{\circ} \mathrm{C}$. The collected supernatants were stored at $-80^{\circ} \mathrm{C}$ for the next step. Total and differential inflammatory cells in BALF were counted using Wright-Giemsa staining (3-5 drops of methanol fixing agent was added to the cells at room temperature for $1 \mathrm{~min}$; Wright-Giemsa stain was then added at room temperature for $8 \mathrm{~min}$, and the cells were finally flushed with running water for 30-60 sec; Wright-Giemsa Stain kit; WGK-1; ScyTek; Shenzhen Xinbosheng Bioengineering Institute) for cytospin preparations.

Immunohistochemical analysis. For immunohistochemistry of AIM2, left lung sections (5- $\mu \mathrm{m}$ thickness) were deparaffinized with xylene, rehydrated in a series of graded concentrations of ethanol and submerged in an antigen repair solution ( $\mathrm{pH}$ 6.0), containing EDTA. All lung tissue sections were treated with $3 \% \mathrm{H}_{2} \mathrm{O}_{2}$ for 20 min to clear away endogenous peroxidase, incubated at polyclonal anti-AIM2 antibody (1:100; cat. no. ab93015; Abcam) at $4^{\circ} \mathrm{C}$ overnight and then, incubated in a horseradish peroxidase (HRP)-linked anti-rabbit secondary antibody (Goat anti-Rabbit IgG (H+L)-HRP; 1:50; cat. no. BS13278; Bioworld Technology, St.) for $30 \mathrm{~min}$. Diaminobenzidine (VECTASTAIN Elite ABC HRP kit; PK-6200; Vector Laboratories) was used to stain the sections (diaminobenzidine staining for $30 \mathrm{~min}$, followed by flushing with running water, staining with hematoxylin for $5 \mathrm{~min}, \mathrm{HCl}$-alcohol differentiation for $20 \mathrm{sec}$, flushing with running water, incubation with $1 \%$ ammonia for $30 \mathrm{sec}$ to return the blue color, dehydration in alcohol and sealing with neutral gum). The process of staining and the following steps were all at room temperature. The IPP 6.0 software (Media Cybernetics, Inc.) was applied for image analysis.

ELISA. The expression levels of TNF- $\alpha$, IL-1 $\beta$, IL-18, IL-6, IL-10 and MCP-1 in BALF and cell culture supernatant were measured using ELISA kits (Mouse TNF- $\alpha$ ELISA kit; cat. no. EK2822/2-96T; Mouse IL-1 $\beta$ ELISA kit; cat. no. EK201B2/2-96T; Mouse IL-18 ELISA kit; cat. no. EK2182-96T; Mouse IL-6 ELISA kit; EK2062/2-96T; Mouse IL-10 ELISA kit; cat. no. EK2012/2-96T; Mouse MCP-1 ELISA kit; cat. no. EK2872/2-96T; MultiSciences), according to the manufacturer's protocol. The concentrations of cytokines were expressed as $\mathrm{pg} / \mathrm{ml}$.

$R N A$ isolation, $c D N A$ synthesis and $R T-q P C R$. Total RNA was extracted from the lower right lung tissues using the TRIzol reagent (Invitrogen; Thermo Fisher Scientific, Inc.) and $1 \mu \mathrm{g}$ RNA was reverse transcribed into cDNA with ReverTra Ace
qPCR RT kit (Toyobo Life Science) (reaction conditions: $37^{\circ} \mathrm{C}$ for $15 \mathrm{~min} ; 98^{\circ} \mathrm{C}$ for $5 \mathrm{~min}$; holding at $4^{\circ} \mathrm{C}$ ). Additionally, RT-qPCR was performed using SYBR Premix Ex Taq ${ }^{\mathrm{TM}}$ kit (Takara Bio Inc.). All the primers were obtained from Sangon Biotech Co., Ltd. The PCR primer sequences are listed in Table I. The amplification was optimized by QuantStudio ${ }^{\mathrm{TM}}$ 6 Flex (Applied Biosystems) using the following PCR procedure: Initial denaturation at $95^{\circ} \mathrm{C}$ for $30 \mathrm{sec}$, with 40 cycles of denaturation at $95^{\circ} \mathrm{C}$ for $5 \mathrm{sec}$, as well as annealing at $60^{\circ} \mathrm{C}$ for $20-30 \mathrm{sec}$. The conditions of melt curve analysis were at $95^{\circ} \mathrm{C}$ for $15 \mathrm{sec}, 59-61^{\circ} \mathrm{C}$ for $60 \mathrm{sec}$ and at $95^{\circ} \mathrm{C}$ for $15 \mathrm{sec}$. Differences in expression levels between the groups were calculated by the $2^{-\Delta \Delta C q}$ method (31) using GAPDH as an internal reference gene.

Western blot analysis. Nuclear and cytoplasmic proteins were extracted from the upper right lung tissue using radioimmunoprecipitation assay lysis buffer (Beyotime Institute of Biotechnology). The method of protein determination was a BCA assay (cat. no. P0010; Beyotime Institute of Biotechnology), and involved calculating the absorbance value at optical density (OD)568 with a DG-3022A enzyme labeling instrument (Nanjing East China Electronics Group Co., Ltd.). The linear regression equation was calculated according to the standard protein concentration and its corresponding OD. According to the OD value of the protein sample, the protein concentration of the sample was calculated via the regression equation. The proteins (40 $\mu \mathrm{g} / \mathrm{lane})$ were resolved on $10 \%$ SDS-PAGE gels by electrophoresis and then transferred onto polyvinylidene difluoride membranes (EMD Millipore) using a Mini Trans-Blot system (Bio-Rad Laboratories Inc.). The membranes were blocked (for $2 \mathrm{~h}$ at room temperature with agitation) with Tris-buffered saline and 20\% Tween-20 containing 5\% non-fat dried milk, and incubated overnight at $4^{\circ} \mathrm{C}$ with antibodies $(1: 1,000)$ against TLR2 (anti-TLR 2 monoclonal; cat. no. 122765; Cell Signaling Technology, Inc.), RAGE (anti-RAGE; cat. no. 6996S; Cell Signaling Technology,

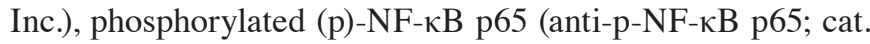
no. 4025S; Cell Signaling Technology, Inc.), ASC (ASC monoclonal antibody; cat. no. 13833S; Cell Signaling Technology, Inc.), AIM2 (anti-AIM2 monoclonal; cat. no. 12948S), TLR4

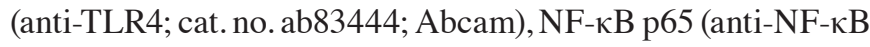
p65; cat. no. ab207297; Abcam) and caspase-1 (anti-caspase-1; cat. no. IMG-5028; Novus Biologicals Ltd.). HRP-conjugated secondary anti-rabbit antibody (1:50,000; cat. no. BM2006; BOSTER Biological Technology Co., Ltd.) was added at $37^{\circ} \mathrm{C}$ for $2 \mathrm{~h}$. Chemiluminescence images were revealed with Pierce ECL Western Blotting Substrate (Thermo Fisher Scientific, Inc.) using BandScan 5.0 gel image software (Glyko, Inc.; Novato) for quantification of the bands.

Generation of bone marrow-derived macrophages. Primary macrophages were prepared from bone marrow progenitors. Bone marrow mononuclear cells were prepared from femur bone marrow suspensions of male C57BL/6 mice (age, 5-8 weeks old) and then cultured in RPMI-1640 medium supplemented with 10\% fetal bovine serum (FBS; HyClone; GE Healthcare Life Science), 0.1\% 100X penicillin-streptomycin solution (Beijing Solarbio Science \& Technology Co., Ltd.) at $37^{\circ} \mathrm{C}$ in a $5 \% \mathrm{CO}_{2}$ incubator. After $4 \mathrm{~h}$, the non-adherent cells were collected. 
Table I. Primer sequences and product sizes.

\begin{tabular}{|c|c|c|}
\hline Gene name & Primer sequences $\left(5^{\prime}-3^{\prime}\right)$ & Product size (bp) \\
\hline TLR2 & & 167 \\
\hline Sense & 5'-CAGTCCCAAAGTCTAAAGTCG-3' & \\
\hline Antisense & 5'-TCTACGGGCAGTGGTGAAA-3' & \\
\hline TLR4 & & 151 \\
\hline Sense & 5'-TGGGTCAAGGAACAGAAGCA-3' & \\
\hline Antisense & 5'-TCACACTGACCACTGACACA-3' & \\
\hline RAGE & & 187 \\
\hline Sense & 5'-CGGGACTCTTTACACTGCG-3' & \\
\hline Antisense & 5'-CCTTCAGGCTCAACCAACA-3' & \\
\hline $\mathrm{NF}-\kappa \mathrm{B}$ & & 112 \\
\hline Sense & 5'-GGACCTATGAGACCTTCAAGAG-3' & \\
\hline Antisense & 5'-ACAGAAGTTGAGTTTCGGGTAG-3' & \\
\hline AIM2 & & 382 \\
\hline Sense & 5'-CACACTCGACGTGGCAGATAGGAC-3' & \\
\hline Antisense & 5'-CAGCACCGTGACAACAAGTGG-3' & \\
\hline ASC & & 121 \\
\hline Sense & 5'-CACCAGCCAAGACAAGATGA-3' & \\
\hline Antisense & 5'-CTCCAGGTCCATCACCAAGT-3' & \\
\hline Caspase-1 & & 190 \\
\hline Sense & 5'-AACAGAACAAAGAAGATGGCACA-3' & \\
\hline Antisense & 5'-CCAACCCTCGGAGAAAGAT-3' & \\
\hline GAPDH & & 150 \\
\hline Sense & 5'-TGTGTCCGTCGTGGATCTGA-3' & \\
\hline Antisense & 5'-TTGCTGTTGAAGTCGCAGGAG-3' & \\
\hline
\end{tabular}

TLR, Toll-like receptor; RAGE, receptor for advanced glycation end products; NF, nuclear factor; ASC, apoptosis-associated speck-like protein containing a CARD.

The adherent cells were suspended $\left(1-2 \times 10^{6}\right.$ cells $\left./ \mathrm{ml}\right)$ in a medium containing $10 \mathrm{ng} / \mathrm{ml}$ macrophage-colony stimulating factor (PeproTech, Inc.) and $10 \mathrm{ng} / \mathrm{ml}$ recombinant mouse IL-4 (PeproTech, Inc.). The medium was replaced on 3rd and 5th days. On 7th day, and the obtained primary macrophages were incubated with or without LPS (100 ng/ml), anti-HMGB1 $(10 \mu \mathrm{g} / \mathrm{ml})(22,32), \mathrm{rHMGB} 1(50 \mu \mathrm{g} / \mathrm{ml})(25,32,33)$, LPS-RS $(1,000 \mathrm{ng} / \mathrm{ml})(25,27)$, or FPS-ZM1 $(1 \mu \mathrm{M})(25,28)$ for $24 \mathrm{~h}$. Macrophages and supernatants were collected for subsequent RT-qPCR, western blotting and ELISA assays.

Flow cytometry. Lung mononuclear cells (MNCs) were re-suspended in FACS buffer (cat. no. 00-4222; eBioscience, Inc.) $\left(2 \times 10^{6}\right.$ cells $\left./ \mathrm{ml}\right)$ and stained with a PE-cluster of differentiation (CD) 11c antibody (cat. no. 561044; eBioscience; Thermo Fisher Scientific, Inc.) and APC-Cy7-F4/80 antibody (cat. no. 123117; BioLegend, Inc.) to characterize the alveolar macrophages (AMs) as CD11c and F4/80 double-positive cells. The antibodies fluorescein isothiocyanate (FITC)-major histocompatibility complex (MHC) II (cat. no. 556643), FITC-CD80 (cat. no. 553768), FITC-CD40 (cat. no. 561845), FITC-CD206 (cat. no. 551135), FITC-IL-10 (cat. no. 554466; eBioscience Inc.) and APC-CD86 (cat. no. 102513; BioLegend,
Inc.) were used to perform the second cell surface staining. All cells were analyzed by flow cytometry (FACSAria III; BD Bioscences). The results were analyzed using FlowJo (version 10.0.7r2; FlowJo LLC)

Statistical analysis. Data are expressed as the mean \pm standard deviation. The differences were compared using the one-way analysis of variance. The post hoc test used was the Bonferroni test. Data were analyzed using the GraphPad Prism 5 software (GraphPad Software, Inc.) and SPSS 22.0 software (IBM, Corps.). $\mathrm{P}<0.05$ was considered to indicate a statistically significant difference. The experiments were performed more than three times.

\section{Results}

HMGB1 aggravates the inflammatory response in $L P S$-induced ALI in a mouse model. To investigate the role of HMGB1 in LPS-induced ALI in a mouse model, anti-HMGB1 and rHMGB1 were injected i.n., $2 \mathrm{~h}$ after LPS, and all mice were sacrificed $24 \mathrm{~h}$ after the last treatment. As shown in Fig. 1A and B, no evident histological alteration of the left lung tissue was observed in the control group and anti-HMGB1 
A

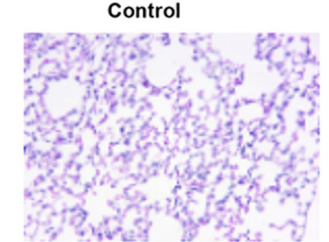

Anti-HMGB1

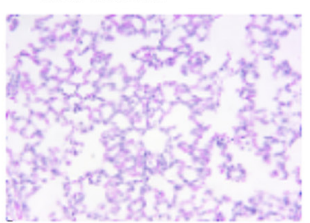

B

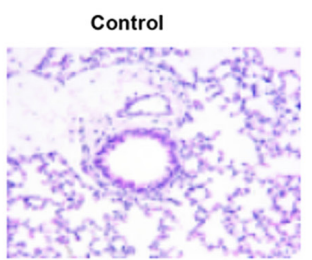

rHMGB1

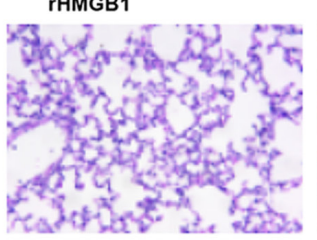

C

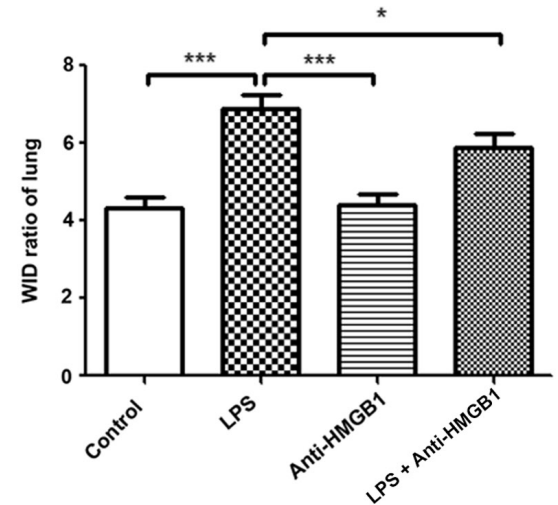

E

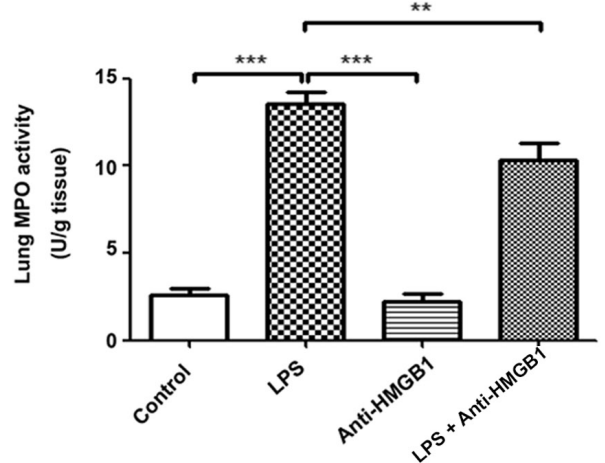

LPS

LPS+Anti-HMGB

LPS

LPS+rHMGB1
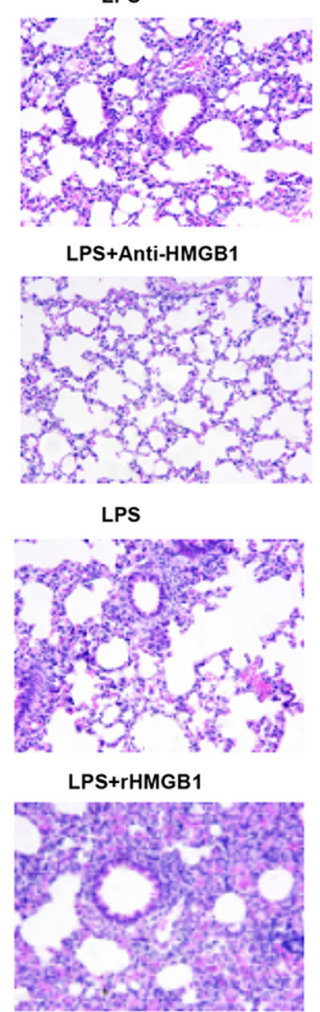

D

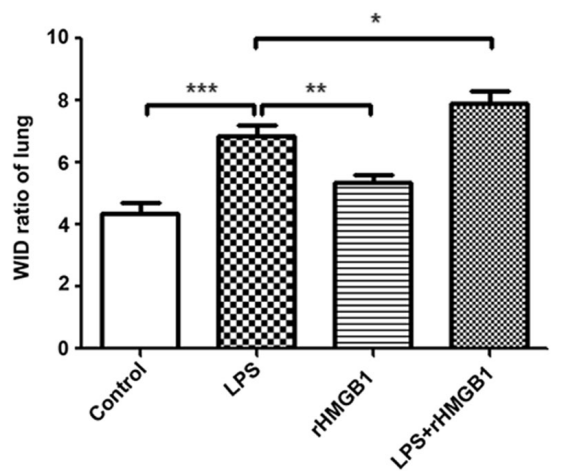

F

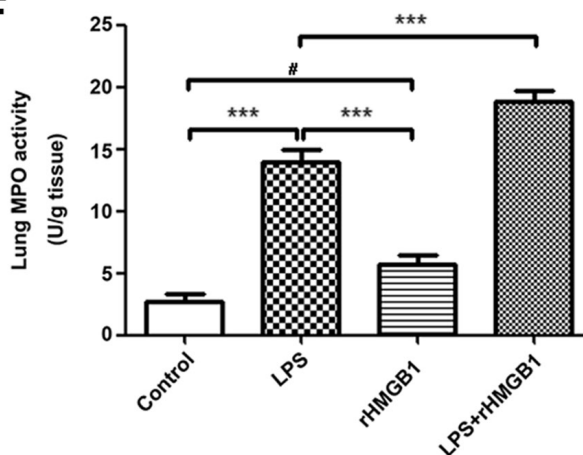

Figure 1. Extracellular HMGB1 affects the inflammatory response in lung tissues in ALI. Histopathological changes and injury scores in the left lung are shown for different groups: (A) Control group, LPS group, Anti-HMGB1 group and LPS + anti-HMGB1 group (H\&E staining, x200 magnification). (B) Control group, LPS group, rHMGB1 group and LPS + rHMGB1 group (H\&E staining, x200 magnification). Effects of (C) anti-HMGB1 and (D) rHMGB1 on the lung W/D ratio of LPS-induced ALI mice. Effects of (E) anti-HMGB1 and (F) rHMGB1 on MPO activity in lung tissues and infiltration of inflammatory cells in BALF of LPS-induced ALI mice. All the experiments were repeated more than three times ( $\mathrm{n}=4-6$ mice per each group). Data presented is from a selected representative experiment. All data are expressed as the mean \pm standard deviation. ${ }^{*} \mathrm{P}<0.05,{ }^{* *} \mathrm{P}<0.01$ and ${ }^{* * *} \mathrm{P}<0.001$ vs. LPS group; ${ }^{*} \mathrm{P}<0.05$ vs. control group.

alone group. However, lung sections of the LPS group showed significant pathological changes, including infiltration of inflammatory cells, edema, hemorrhage and alveolar collapse
$(\mathrm{P}<0.001)$. Slight pathological changes were observed in the rHMGB1 alone group. Treatment with anti-HMGB1 markedly ameliorated LPS-induced lung injury and administration of 

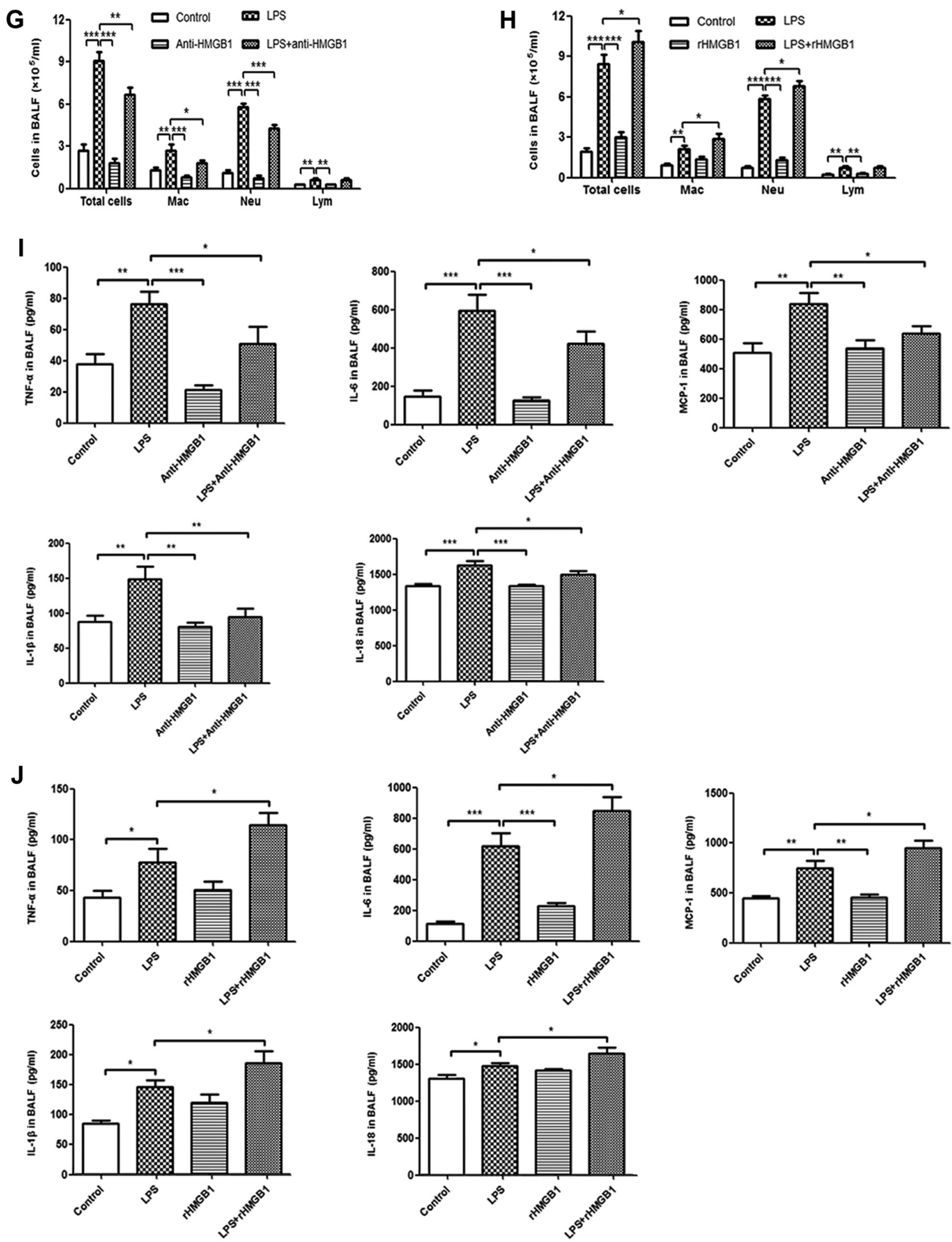

Figure 1. Continued. Extracellular HMGB1 affects the inflammatory response in lung tissues in ALI. Effects of (G) anti-HMGB1 and (H) rHMGB1 on MPO activity in lung tissues and infiltration of inflammatory cells in BALF of LPS-induced ALI mice. Effects of (I) anti-HMGB1 and (J) rHMGB1 on the ELISA results of secretion of inflammatory cytokines (TNF- $\alpha$, IL-6, MCP-1, IL-1 $\beta$, and IL-18) in BALF. All the experiments were repeated more than three times ( $\mathrm{n}=4-6$ mice per each group). Data presented is from a selected representative experiment. All data are expressed as the mean \pm standard deviation. ${ }^{*} \mathrm{P}<0.05$, ${ }^{* *} \mathrm{P}<0.01$ and ${ }^{* * * *} \mathrm{P}<0.001$ vs. LPS group; ${ }^{*} \mathrm{P}<0.05$ vs. control group. LPS, lipopolysaccharide; IL, interleukin; BALF, bronchoalveolar lavage fluid; MCP, myeloperoxidase; TNF, tumor necrosis factor; ALI, acute lung injury; rHMGB1, recombinant High mobility group box 1; H\&E, hematoxylin and eosin; W/D, wet to dry.

rHMGB1 significantly aggravated the pathological injury of lung tissue $(\mathrm{P}<0.001)$. The lung $\mathrm{W} / \mathrm{D}$ ratio in lung tissues was notably decreased in the LPS challenge group compared with that of the control group. There were no significant differences 
A

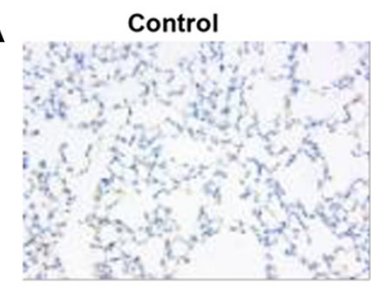

Anti-HMGB1

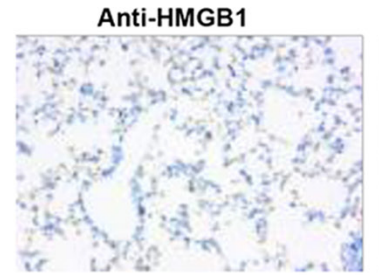

B

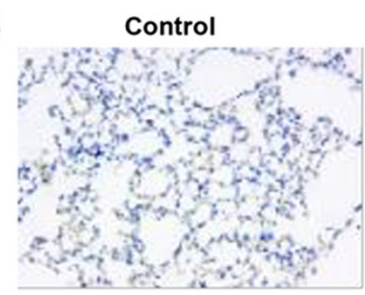

rHMGB1

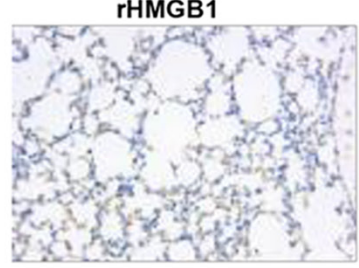

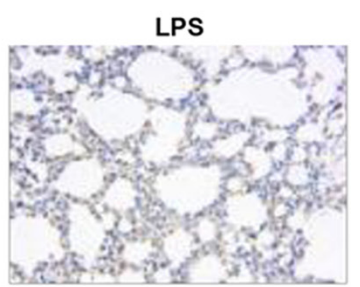

LPS+Anti-HMGB1

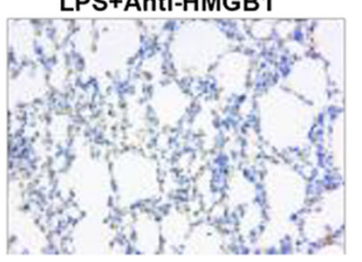

LPS

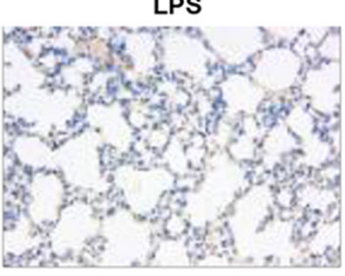

LPS+rHMGB1

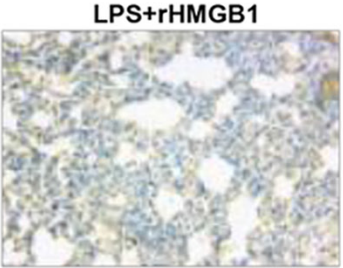

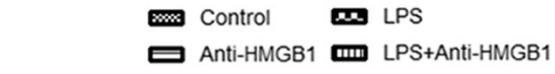
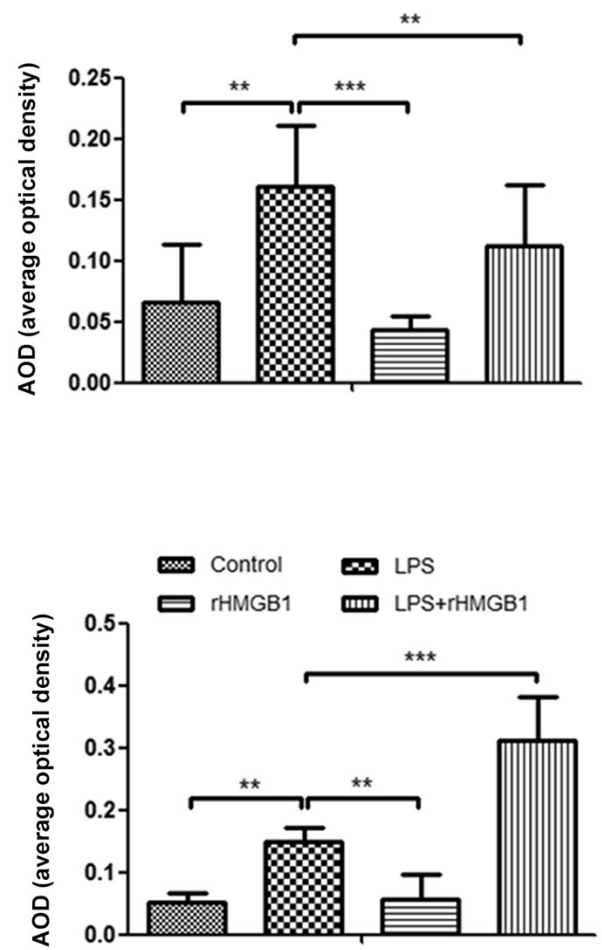
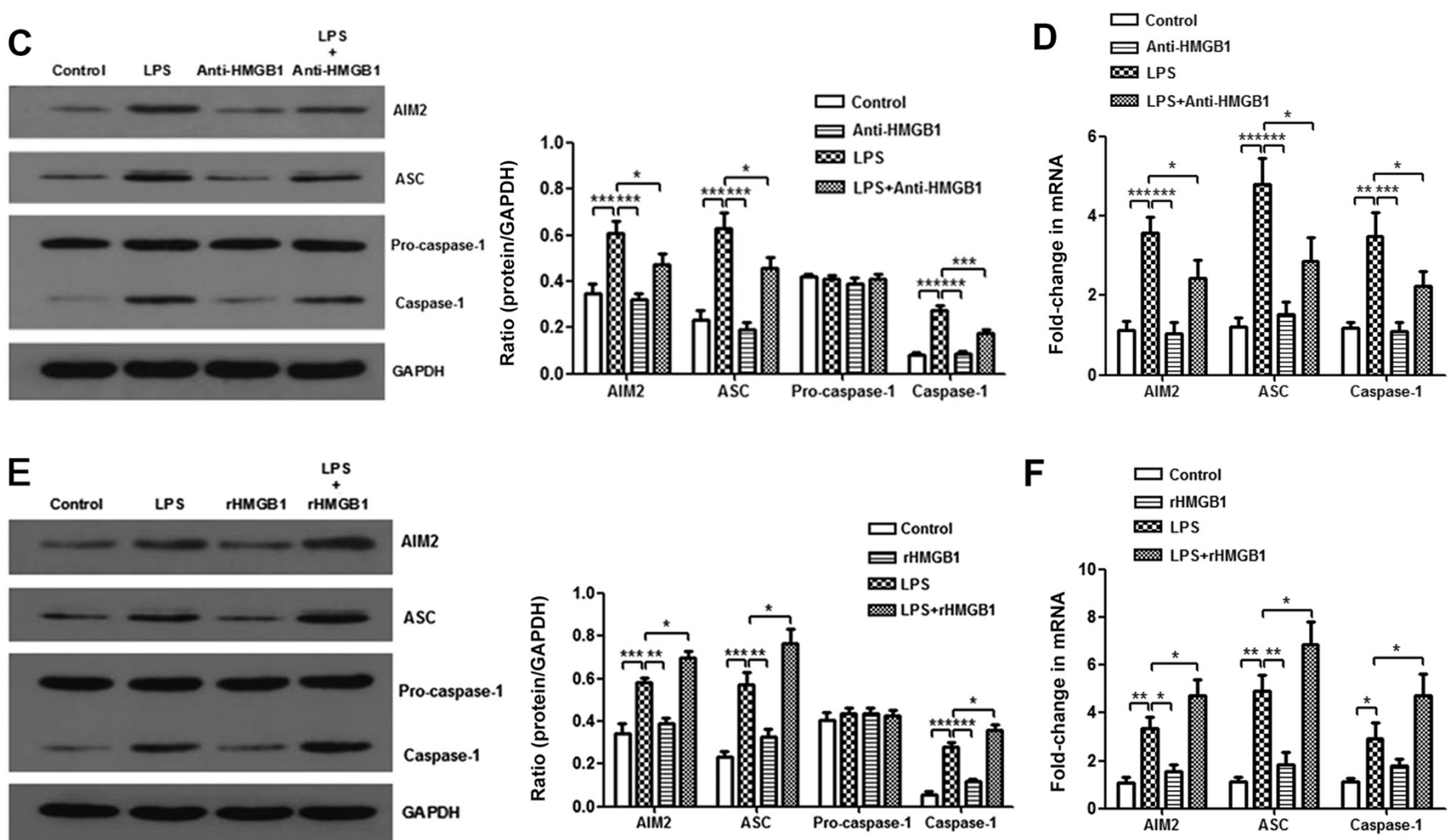

Figure 2. Expression level of AIM2 inflammasome is upregulated by HMGB1. Effects of (A) anti-HMGB1 and (B) rHMGB1 on the expression level of AIM2 in mouse lung tissue was detected by immunohistochemistry (magnification, $\mathrm{x} 200$ ), and AOD was analyzed in different groups. In the in vivo experiment, the expression levels of AIM2 inflammasome and GAPDH were detected by (C and E) western blotting with (C) anti-HMGB1 and (E) rHMGB1 and RT-qPCR with (D) anti-HMGB1 and (F) rHMGB1. All experiments were repeated more than three times (n=4-6 mice per each group). Data presented is from a representative experiment. All data are expressed as the mean \pm standard deviation. ${ }^{*} \mathrm{P}<0.05,{ }^{* *} \mathrm{P}<0.01$ and ${ }^{* * *} \mathrm{P}<0.001$ vs. LPS group. 
G

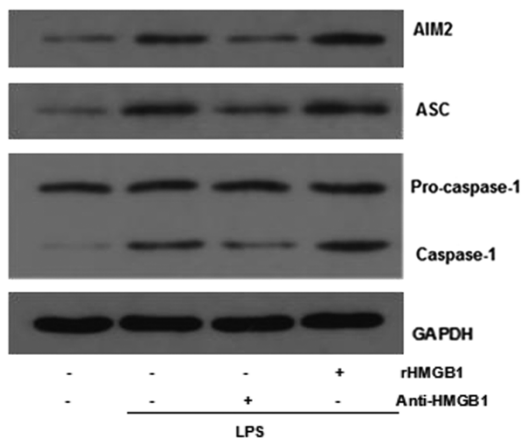

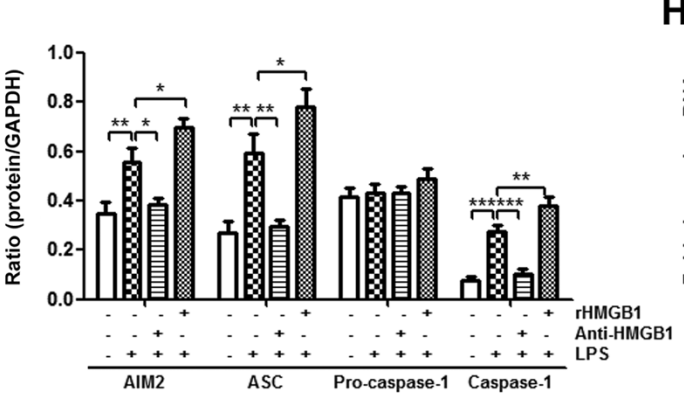

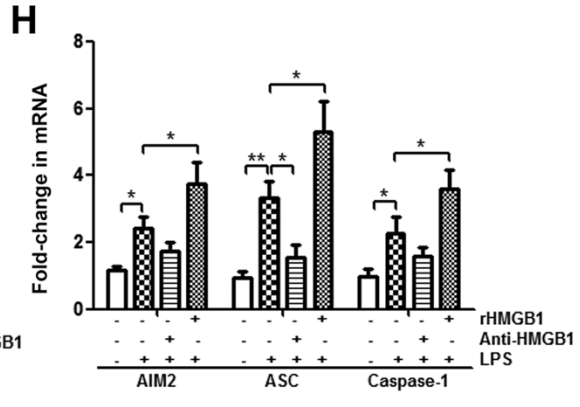

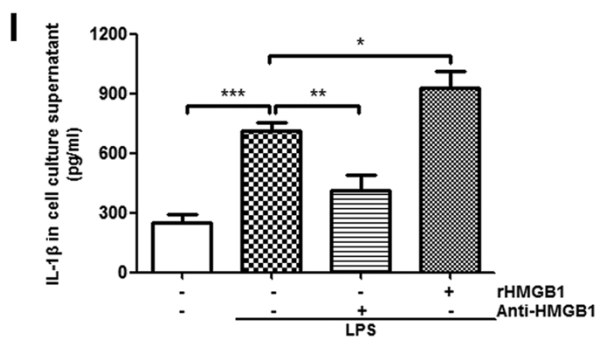

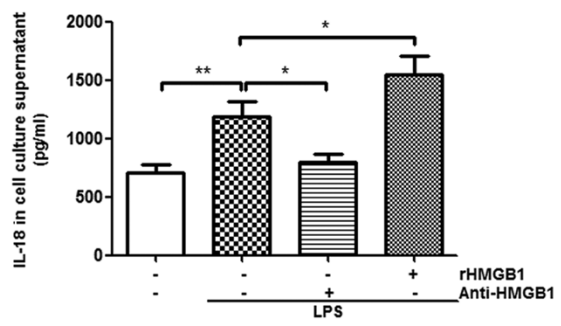

Figure 2. Continued. Expression level of AIM2 inflammasome is upregulated by HMGB1. In an experiment with BMMs, the expression levels of the AIM2 inflammasome and GAPDH were also detected by (G) western blotting and (H) RT-qPCR. (I) The expression levels of IL-1 $\beta$ and IL-18 in culture supernatant of BMMs were measured by ELISA. All experiments were repeated more than three times ( $n=4-6$ mice per each group). Data presented is from a representative experiment. All data are expressed as the mean \pm standard deviation. ${ }^{*} \mathrm{P}<0.05,{ }^{* * *} \mathrm{P}<0.01$ and ${ }^{* * *} \mathrm{P}<0.001$ vs. LPS group. LPS, lipopolysaccharide; IL, interleukin; rHMGB1, recombinant High mobility group box 1; RT-q, reverse transcription-quantitative; AIM2, absent in melanoma 2; AOD, average optical density; BMMs, bone-marrow derived macrophages; ASC, apoptosis-associated speck-like protein containing a CARD.

of W/D ratio in lung tissues between anti-HMGB1, rHMGB1 alone and control groups. Treatment with anti-HMGB1 significantly decreased the W/D ratio in lung tissues and rHMGB1 significantly increased that ratio $(\mathrm{P}<0.001$; Fig. $1 \mathrm{C}$ and $\mathrm{D})$. As neutrophils are the main cells involved in inflammatory reactions in injured lungs, MPO activity was measured to reflect neutrophil accumulation in lung tissues. In the present study, LPS and single rHMGB1 administration significantly increased MPO activity compared with the control group $(\mathrm{P}<0.001$; Fig. 1E and F). Treatment with anti-HMGB1 significantly decreased MPO activity in lung tissues of LPS-induced ALI mice, while rHMGB1 had the opposite effect $(\mathrm{P}<0.001$; Fig. 1E and F). The numbers of inflammatory cells to evaluate pulmonary inflammation were also examined. After LPS challenge, the numbers of total cells, macrophages, neutrophils and lymphocytes significantly increased compared with those of the control group $(\mathrm{P}<0.001)$. This increase in total cells, macrophages and neutrophils was significantly attenuated by treatment with anti-HMGB1 and aggravated by rHMGB1 ( $\mathrm{P}<0.01$; Fig. $1 \mathrm{G}$ and H). The levels of TNF- $\alpha$, IL-6, MCP-1, IL-1 $\beta$ and IL-18 production in BALF were detected by ELISA. The results showed that their concentrations were significantly increased in the LPS-challenged mice compared with in the mice of the control group $(\mathrm{P}<0.05)$. Treatment with anti-HMGB1 after LPS challenge significantly inhibited the proinflammatory cytokine production $(\mathrm{P}<0.05)$ and treatment with rHMGB1 increased their levels (Fig. 1I and J). These results suggest that HMGB1 may aggravate the inflammatory response in LPS-induced ALI.

HMGB1 activates the AIM2 inflammasome complex in macrophages. To further explore the mechanism of HMGB1 in ALI, the present study attempted to study the relationship between HMGB1 and the AIM2 inflammasome. AIM2 was immunostained with anti-AIM2 to further determine AIM2 expression in lung tissue sections. It was observed that AIM2 was mainly expressed in inflammatory cells, such as macrophages and neutrophils. There were more positively stained cells in lung tissues of the LPS+rHMGB1 group compared with those of the LPS group, whereas there were fewer positively stained cells in the LPS+anti-HMGB1 group (Fig. 2A and B). In the in vivo experiment, LPS significantly upregulated the expression levels of AIM2, ASC and caspase-1, except for pro-caspase-1, which is an inactive precursor of caspase-1, as determined by western blot analysis $(\mathrm{P}<0.001)$. This increase was aggravated by rHMGB1 administration; however, anti-HMGB1 inhibited expression of LPS-induced the AIM2 inflammasome (Fig. 2C and E). Similar results were obtained by RT-qPCR detection of AIM2, ASC and caspase-1 in lung tissues (Fig. 2D and F). To further study their relationships at the macrophage level, bone marrow-derived macrophages (BMMs) primed with LPS and treated with anti-HMGB1 or rHMGB1 were cultured. The expression level of the inflammasome in BMMs was detected by western blotting and RT-qPCR. As illustrated in Fig. 2G and H, the expression levels of AIM2, ASC and caspase-1 proteins significantly increased in the LPS group, and the significant increase was greater in the LPS+rHMGB1 group $(\mathrm{P}<0.05)$. In the LPS+anti-HMGB1 group, ASC showed a significant decrease compared with the LPS group (Fig. 2H), although a significant decrease in expression levels of AIM2, ASC and caspase-1 was observed in Fig. 2G. The activated AIM2 inflammasome induces pro-IL-1 $\beta$ and pro-IL-18 cleavage into active IL-1 $\beta$ and IL-18. That is to say, IL-1 $\beta$ and IL-18 in the culture supernatant are 
A
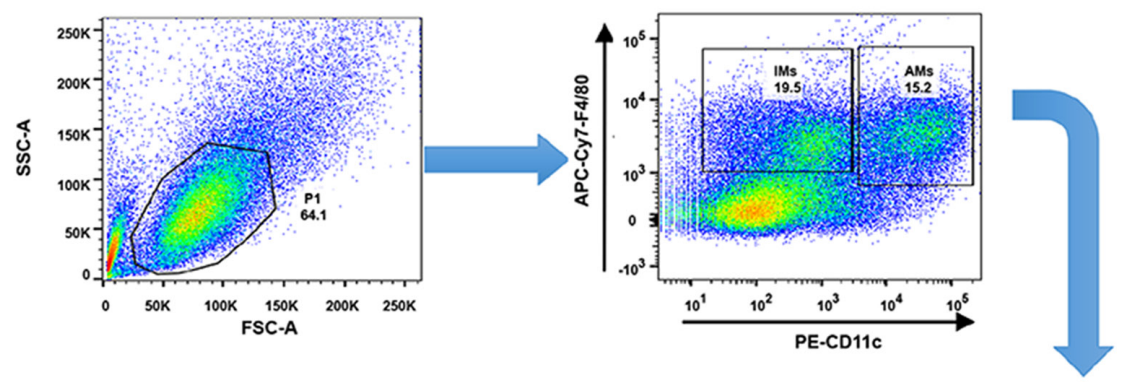

B
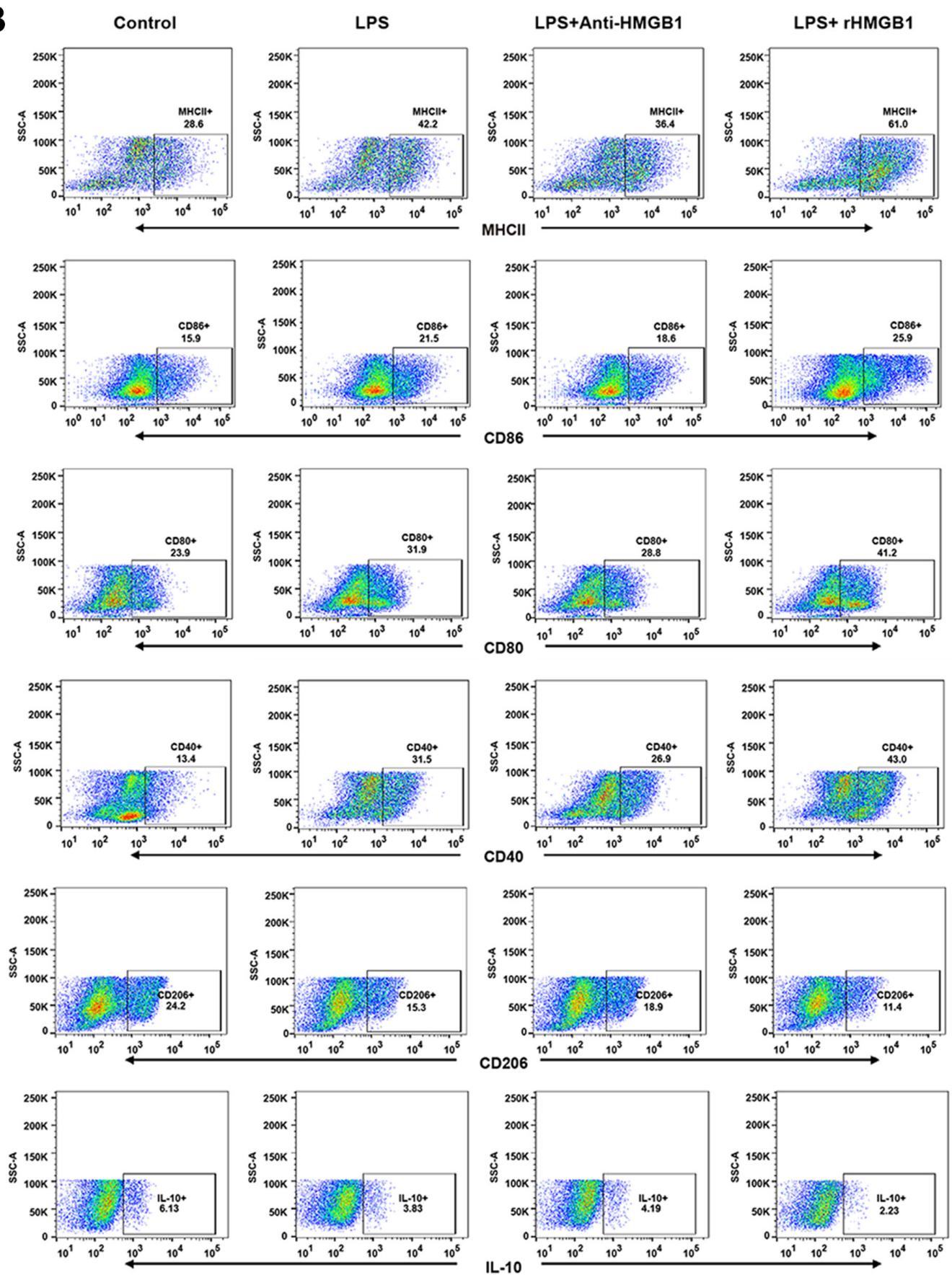

Figure 3. Upregulation surface markers and cytokines of M1 macrophages by extracellular HMGB1 (A) Flow cytometry was used to identify F4/80+ and

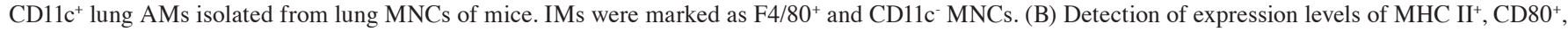
$\mathrm{CD} 6^{+}, \mathrm{CD} 40^{+}, \mathrm{CD} 206^{+}$and IL-10 on AM cell surface. All experiments were repeated more than three times (n=4-6 mice per each group). Data presented is from a representative experiment. All data are expressed as the mean \pm standard deviation.

downstream of the AIM2 inflammasome in BMMs. They could indirectly reflect activation of the AIM2 inflammasome in macrophages. As illustrated in Fig. 2I, the concentrations of IL-1 $\beta$ and IL-18 in culture supernatants were significantly 

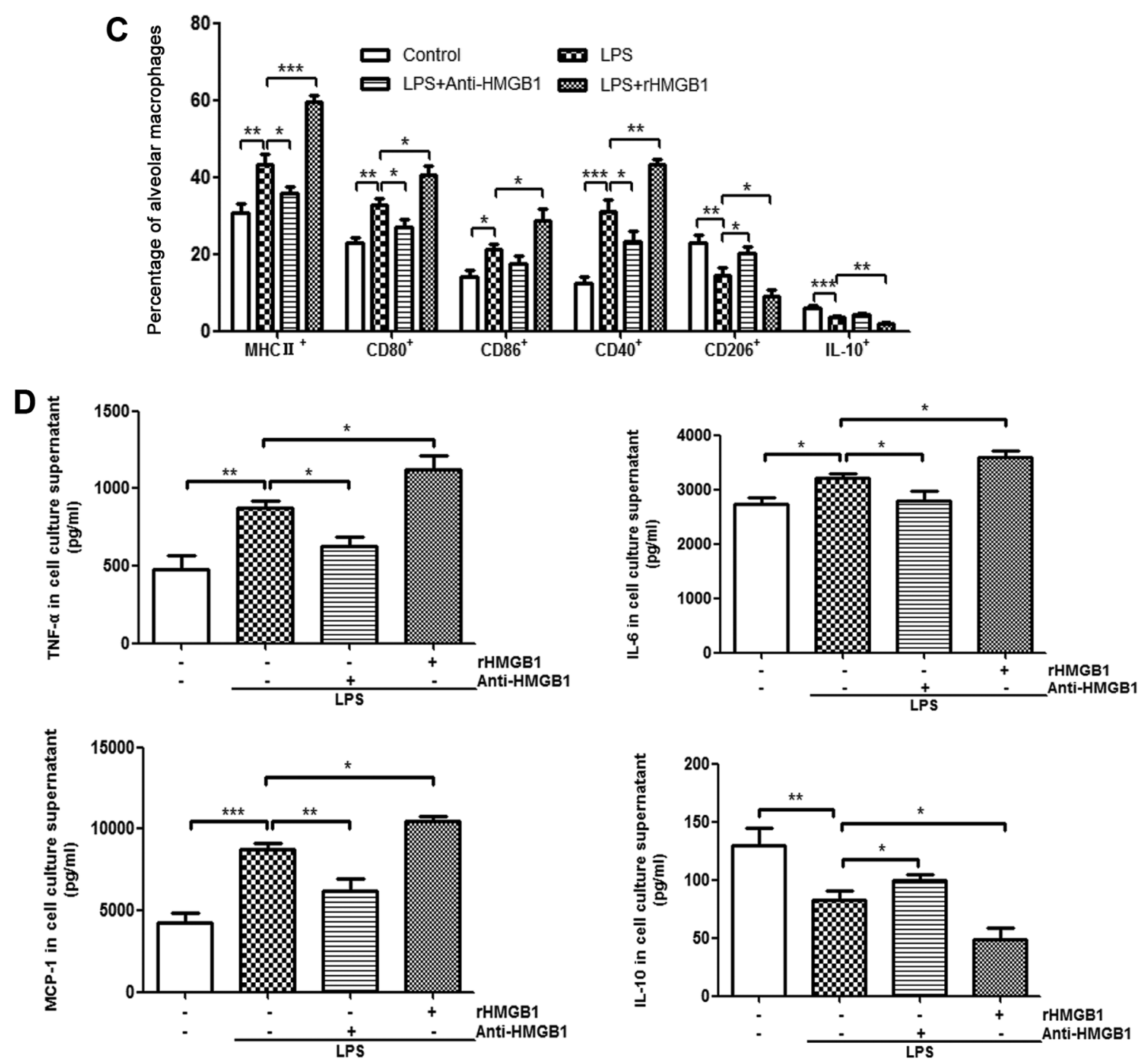

Figure 3. Continued. Upregulation surface markers and cytokines of M1 macrophages by extracellular HMGB1. (C) The percentages of lung AMs expressing MHC II, CD80, CD86, CD40, CD206 and IL-10 were calculated. (D) AM activation is defined by two distinct polarization states: M1 and M2. M1-related cytokines (tumor necrosis factor- $\alpha$, IL-6, and MCP-1) and M2-related cytokine (IL-10) were detected in culture supernatant of BMMs by ELISA. All experiments were repeated more than three times $(n=4-6$ mice per each group). Data presented is from a representative experiment. All data are expressed as the mean \pm standard deviation. " $\mathrm{P}<0.05,{ }^{* *} \mathrm{P}<0.01$ and ${ }^{* * *} \mathrm{P}<0.001$ vs. LPS group. LPS, lipopolysaccharide; IL, interleukin; MCP, myeloperoxidase; rHMGB1, recombinant High mobility group box 1; CD, cluster of differentiation; MHC, major histocompatibility complex; AMs, alveolar macrophages; MNCs, mononuclear cells; BMMs, bone-marrow derived macrophages.

increased in LPS-primed groups $(\mathrm{P}<0.01)$, with a maximum increase in the rHMGB1 group and minimum elevation in the anti-HMGB1 group. These results suggest that HMGB1 may activate the AIM2 inflammasome in macrophages, accelerating infiltration of inflammatory cells and increasing the level of its downstream inflammatory cytokines in LPS-induced ALI.

HMGB1 induces polarization of M1 macrophages. AMs form the first line of defense in the inflammatory response and phagocytosis of pathogens. A previous study demonstrated that M1 AMs have been implicated in the pathogenesis of ALI and M2 AMs were mainly described as having anti-inflammatory or reparative functions (34). To explore whether HMGB1 had a relationship with AMs in an LPS-induced ALI mouse model, the present study attempted to investigate whether HMGB1 regulated polarization of $\mathrm{M} 1$ or $\mathrm{M} 2$ macrophages and the function of AMs. Lung interstitial macrophages (IMs) express the macrophage marker F4/80, rather than the marker CD11c, whereas lung AMs are F4/80 and CD11c double-positive MNCs $(35,36)$. Lung MNCs were subsequently stained with F4/80-APC-Cy7 and CD11c-PE, and analyzed by flow cytometry. It was found that the lung $\mathrm{F} 4 / 80^{+}$macrophages were divided into two subgroups (IMs and AMs; Fig. 3A). M1-related surface markers (MHC II, CD80, CD86 and CD40) and M2-related markers (CD206 and IL-10) from the gating region of lung $\mathrm{AMs}\left(\mathrm{F} 4 / 80^{+} \mathrm{CD} 11 \mathrm{c}^{+}\right)$were then observed. The percentage of MHC II, CD80, CD86 and CD40-expressing AMs was significantly increased in ALI mice compared with in control mice. It was also noted that their percentages further increased in the ALI group that was administered rHMGB1 and decreased when treated with anti-HMGB1. However, the percentage of CD206, IL-10-expressing AMs showed an antipodal tendency (Fig. 3B and C). As described 
A

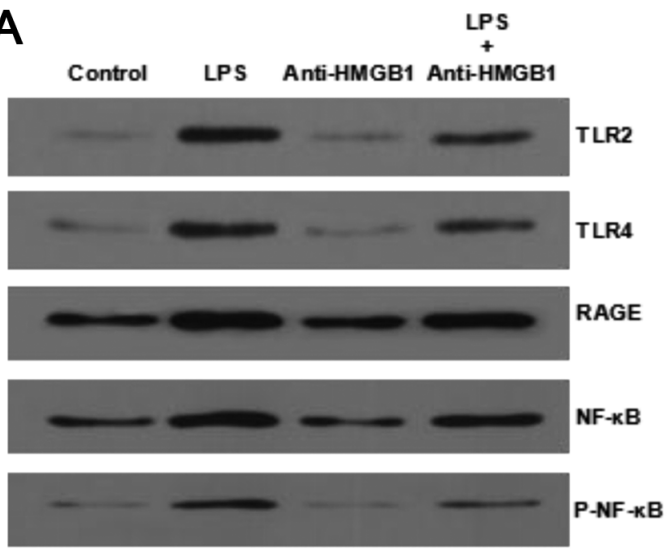

20 GAPDH

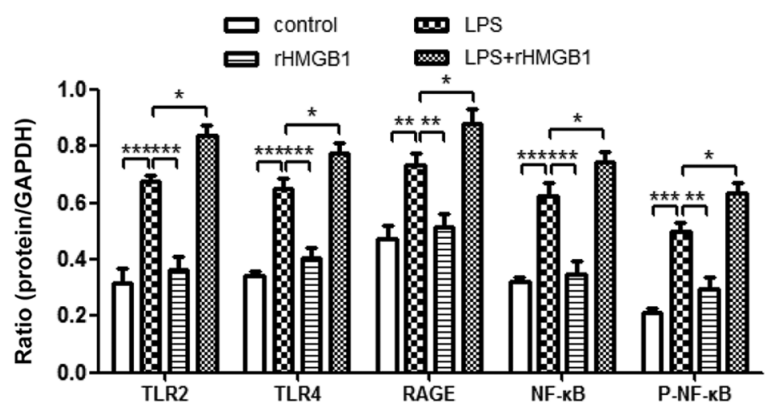

C
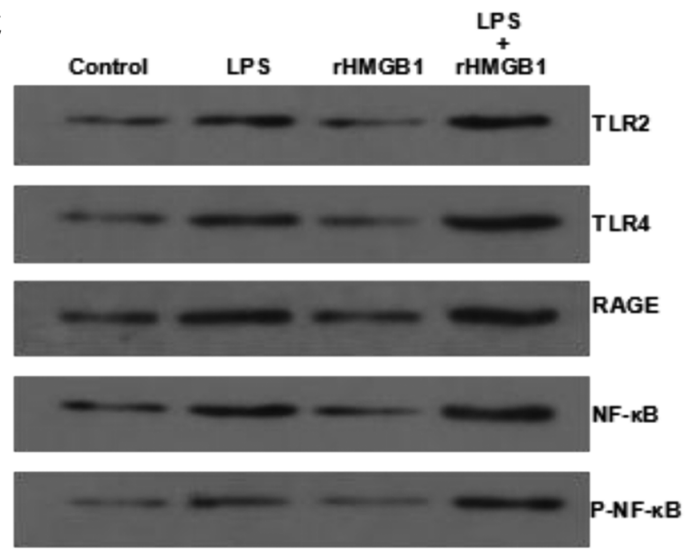

GAPDH

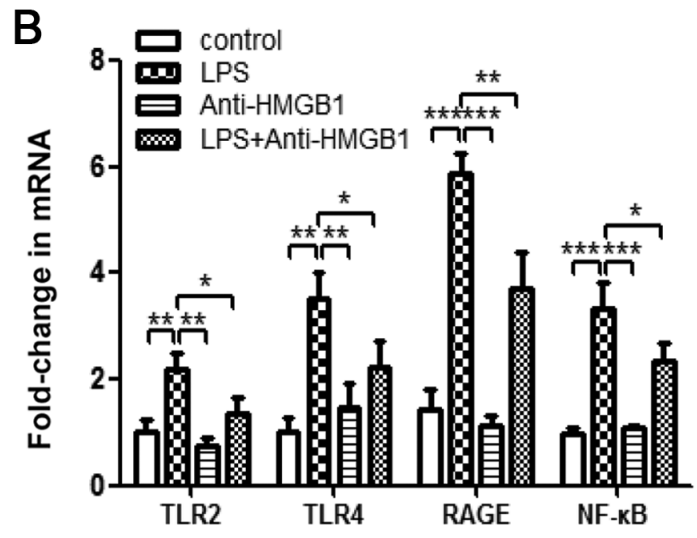

D

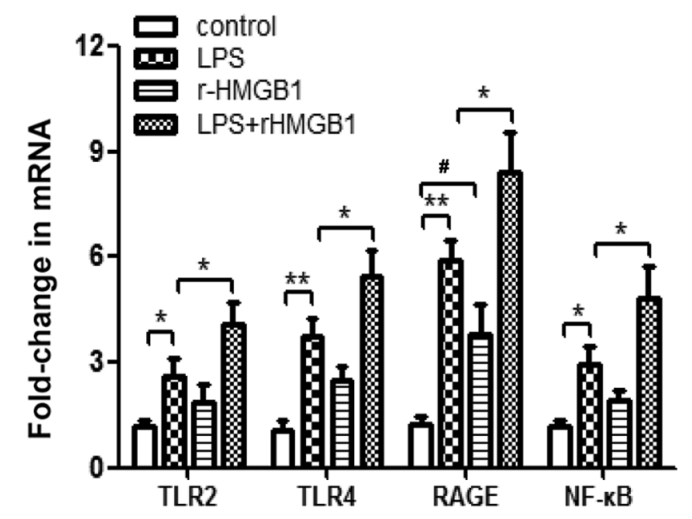

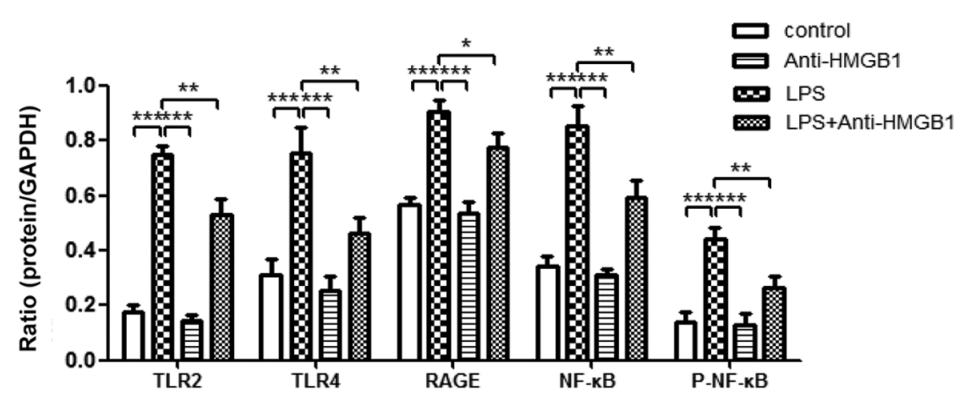

Figure 4. Enhanced expression levels of TLR2, TLR4, RAGE and NF- $x$ B in macrophages by extracellular HMGB1. Expression levels of TLR2, TLR4, RAGE,

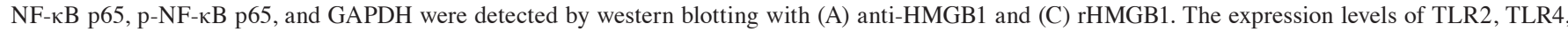
RAGE, NF- $\kappa$ B, and GAPDH were detected by RT-qPCR with (B) anti-HMGB1 and (D) rHMGB1 in the right lungs of LPS-induced ALI mice and control mice. All experiments were repeated more than three times $(n=4-6$ mice per each group). Data presented is from a representative experiment. All data are expressed as the mean \pm standard deviation. ${ }^{*} \mathrm{P}<0.05,{ }^{* *} \mathrm{P}<0.01$ and ${ }^{* * *} \mathrm{P}<0.001$ vs. LPS group. ${ }^{\#} \mathrm{P}<0.05$ vs. control group. 

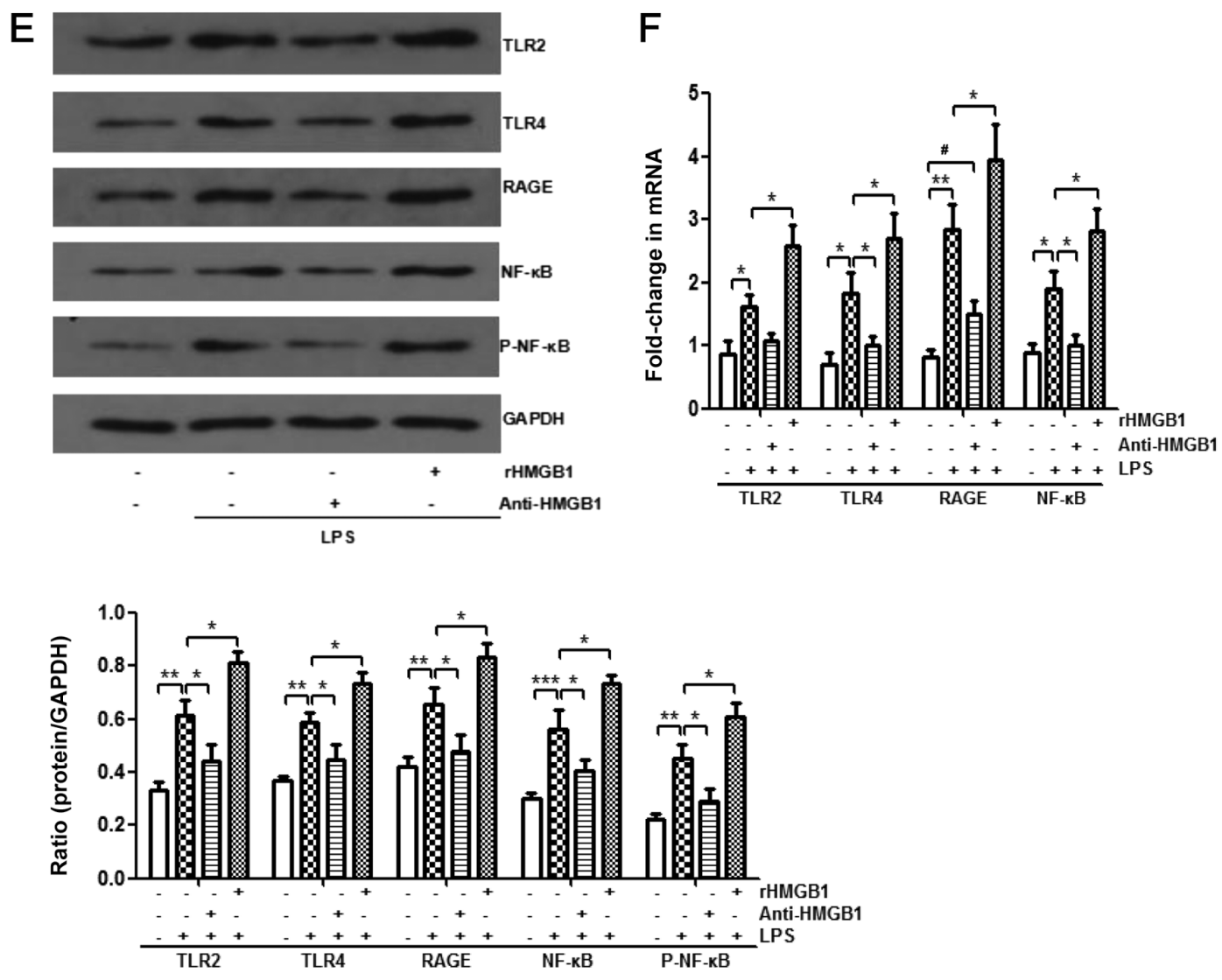

Figure 4. Continued. Enhanced expression levels of TLR2, TLR4, RAGE and NF- $\mathrm{kB}$ in macrophages by extracellular HMGB1. The expression levels of TLR2, TLR4, RAGE, NF- $\mathrm{kB}$, and GAPDH were detected by RT-qPCR with anti-HMGB1 and rHMGB1 in the right lungs of LPS-induced ALI mice and control mice, and in LPS-primed BMMs stimulated with (E) anti-HMGB1 or (F) rHMGB1. All experiments were repeated more than three times (n=4-6 mice per each group). Data presented is from a representative experiment. All data are expressed as the mean \pm standard deviation. ${ }^{*} \mathrm{P}<0.05,{ }^{* *} \mathrm{P}<0.01$ and ${ }^{* * * *} \mathrm{P}<0.001$ vs. LPS group. ${ }^{*} \mathrm{P}<0.05$ vs. control group. LPS, lipopolysaccharide; IL, interleukin; MCP, myeloperoxidase; TNF, tumor necrosis factor; ALI, acute lung injury; rHMGB1, recombinant High mobility group box 1; TLR, toll-like receptor; RAGE, receptor for advanced glycation end products; RT-q, reverse transcription-quantitative; p-NF, phosphorylated-nuclear factor; BMMs, bone-marrow derived macrophages.

above, HMGB1 may mediate polarization of M1 macrophages in the ALI mouse model. In vitro, it was also observed that HMGB1 regulated polarization of M1 macrophages by detecting the M1-related cytokines (TNF- $\alpha$, IL-6 and MCP-1) and M2-related cytokine (IL-10) in the culture supernatant of BMMs stimulated with anti-HMGB1 or rHMGB1 by ELISA. The results showed that the expression levels of TNF- $\alpha$, IL-6 and MCP-1 increased, while the expression level of IL-10 decreased in LPS-stimulated group compared with those in the control group. Moreover, the changes were significantly enhanced by rHMGB1 stimulation $(\mathrm{P}<0.05)$, however they were weakened by anti-HMGB1 stimulation (Fig. 3D). Therefore, HMGB1 can induce polarization of M1 macrophages in vivo and in vitro.

HMGB1 acts on macrophages through TLR2, TLR4 and $R A G E / N F-\kappa B$ signaling pathways. HMGB1 stimulates the inflammatory response through several pattern-recognition receptors, including RAGE, TLR2 and TLR4 $(5,25,37,38)$. HMGB1 interacts with those receptors by activating the $\mathrm{NF}-\kappa \mathrm{B}$ signaling pathway and downstream inflammatory mediators $(5,39,40)$. However, whether HMGB1 acts on macrophages through TLR2, TLR4 and RAGE/NF- $\kappa$ B signaling pathways in ALI needs to be further verified. Treatment with anti-HMGB1 was found to inhibit upregula- tion of expression levels of LPS-induced TLR2, TLR4, RAGE, $\mathrm{NF}-\kappa \mathrm{B}$ p 65 and $\mathrm{p}-\mathrm{NF}-\kappa \mathrm{B}$ p 65 in the animal experiments by western blot analysis (Fig. 4A). Whether anti-HMGB1 affected mRNA expression of TLR2, TLR4, RAGE and NF- $\mathrm{B}$ was also examined. As displayed in Fig. 4B, the same inhibiting effect was observed. In addition, administration of rHMGB1 enhanced expression levels of LPS-induced TLR2, TLR4, RAGE, NF- $\kappa$ B p65 and p-NF- $\kappa$ B p65 proteins (Fig. 4C), as well as expression levels of TLR2, TLR4, RAGE, and NF- $\kappa \mathrm{B}$ mRNA (Fig. 4D). Western blotting and RT-qPCR were also used to analyze LPS-primed BMMs stimulated with anti-HMGB1 or rHMGB1. As shown in Fig. 4E, administration of LPS significantly increased the expression levels of

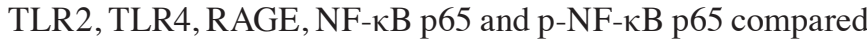
with the control group $(\mathrm{P}<0.01)$. However, stimulation of anti-HMGB1 significantly decreased their expression levels induced by LPS $(\mathrm{P}<0.05)$ and the increases were weakened upon rHMGB1 stimulation. Similar to the present findings by western blot analysis, the expression levels of TLR2, TLR4, RAGE and NF- $\kappa \mathrm{B}$ mRNA were notably increased in BMMs stimulated with LPS. However, these increases were markedly inhibited by treatment with anti-HMGB1 and significantly promoted by treatment with rHMGB1 ( $\mathrm{P}<0.05$; Fig. 4F). Thus, HMGB1 might act on macrophages through TLR2, TLR4 and $\mathrm{RAGE} / \mathrm{NF}-\kappa \mathrm{B}$ signaling pathways. 
A

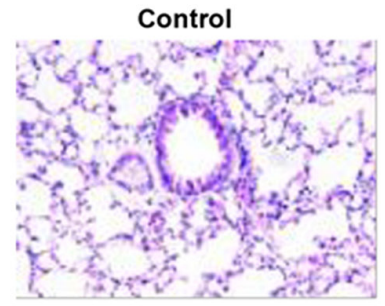

LPS-RS

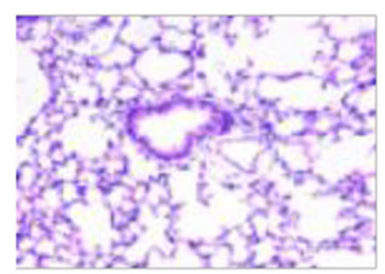

B

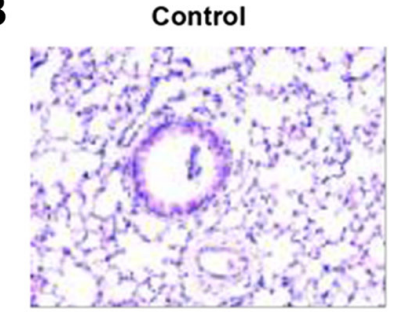

FPS-ZM1
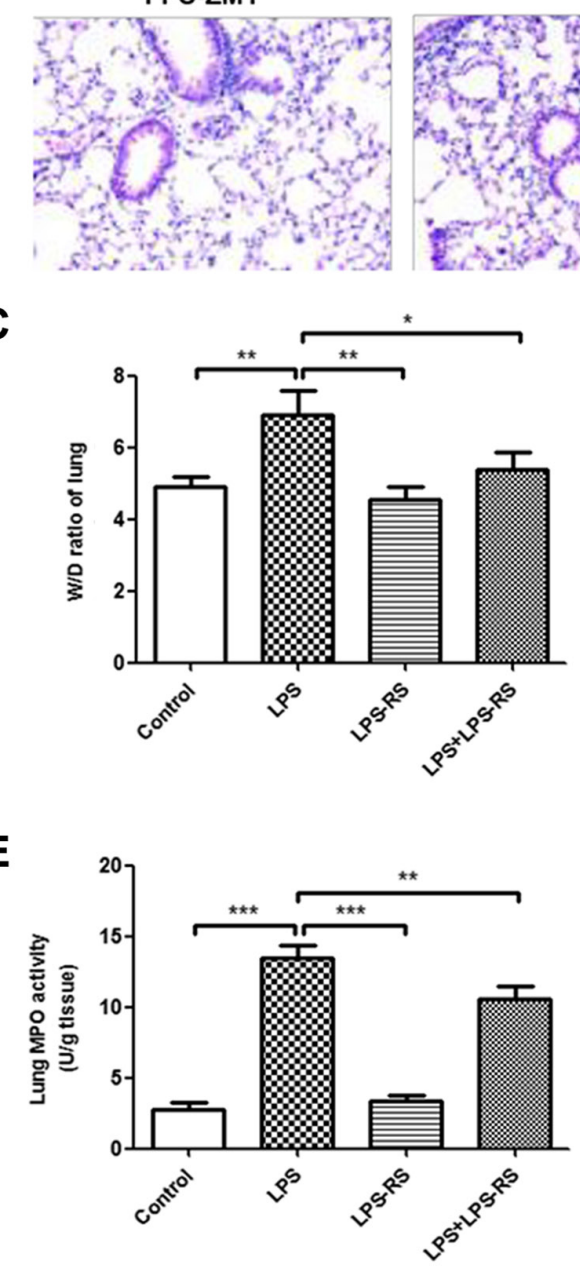

LPS

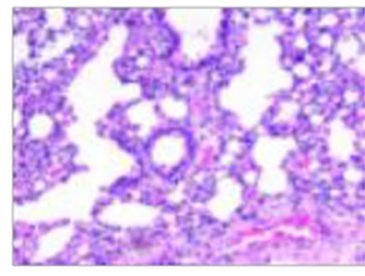

LPS+LPS-RS

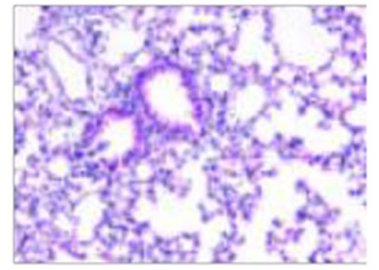

LPS

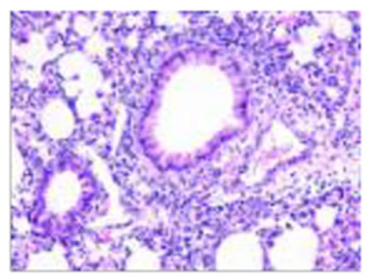

LPS+FPS-ZM1

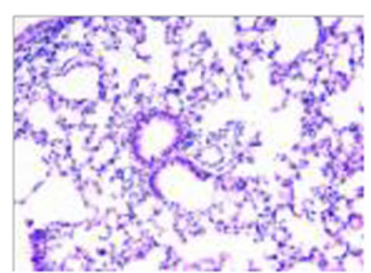

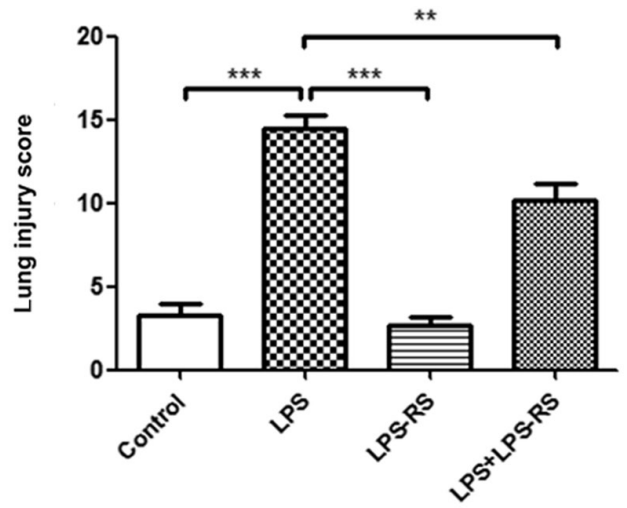

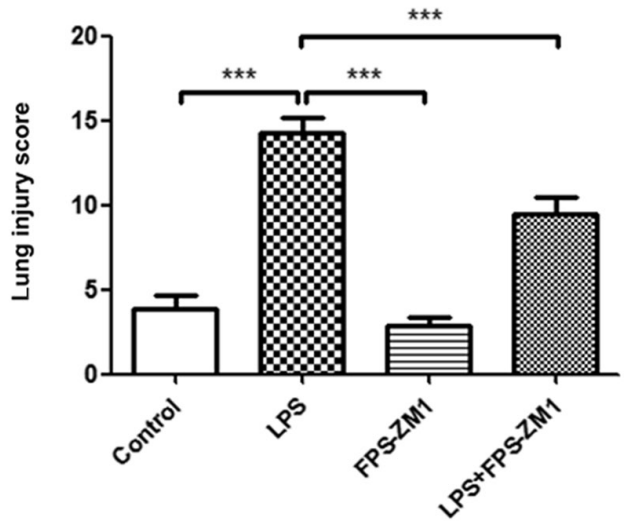

C

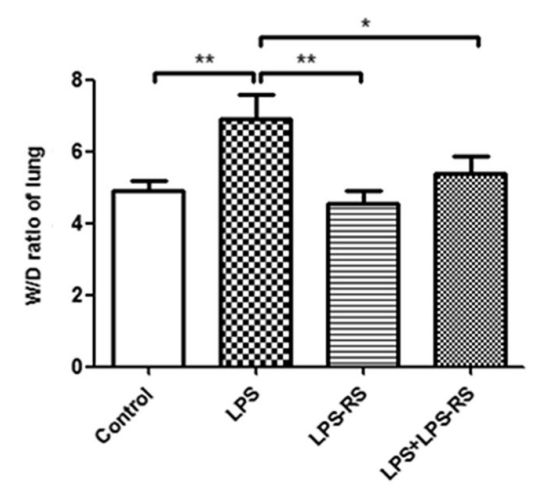

E

D

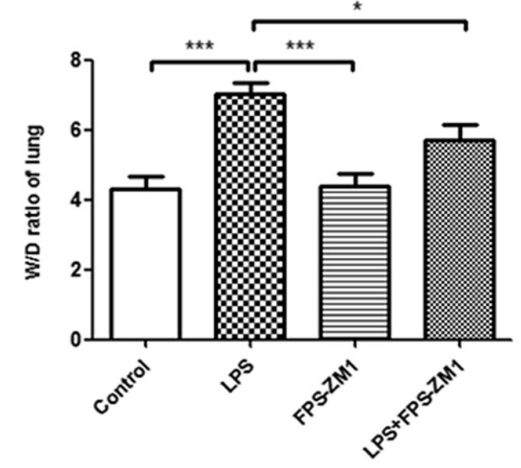

F

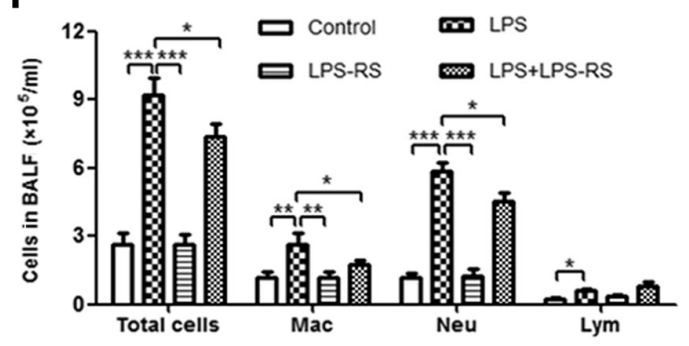

Figure 5. Reduced inflammatory response in lung tissue in ALI by administration of TLR2/4 and RAGE antagonists. Histopathological changes and injury scores in left lung are illustrated for different groups: (A) Control group, LPS group, LPS-RS group, and LPS + LPS-RS group (H\&E staining, x200). (B) Control group, LPS group, FPS-ZM1 group and D: LPS+FPS-ZM1 group (H\&E staining, x200). Effects of (C) LPS-RS and (D) FPS-ZM1 on the lung W/D ratio of LPS-induced ALI mice. Effects of LPS-RS and on (E) MPO activity in lung tissues and (F) infiltration of inflammatory cells in BALF of LPS-induced ALI mice. All experiments were repeated more than three times ( $\mathrm{n}=4-6$ mice per each group). Data presented is from a representative experiment. All data are expressed as the mean \pm standard deviation. ${ }^{*} \mathrm{P}<0.05,{ }^{* * *} \mathrm{P}<0.01$ and ${ }^{* * *} \mathrm{P}<0.001$ vs. LPS group. 

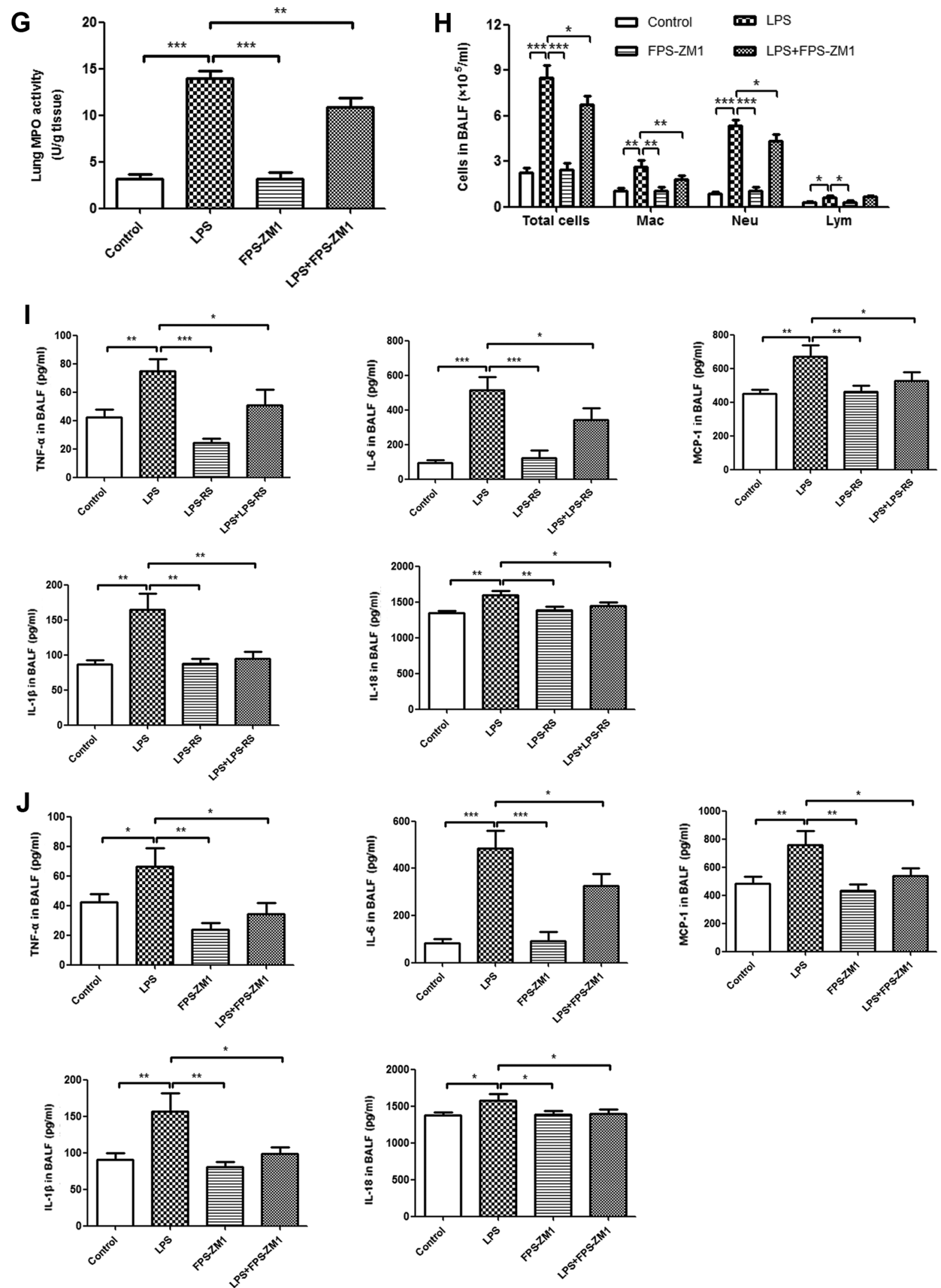

Figure 5. Continued. Reduced inflammatory response in lung tissue in ALI by administration of TLR2/4 and RAGE antagonists. Effects of FPS-ZM1 on (G) MPO activity in lung tissues and (H) infiltration of inflammatory cells in BALF of LPS-induced ALI mice. ELISA results of secretion of inflammatory cytokines (TNF- $\alpha$, IL-6, MCP-1, IL-1 $\beta$ and IL-18) treated with (I) LPS-RS and (J) FPS-ZM1 in BALF. All experiments were repeated more than three times ( $\mathrm{n}=4-6$ mice per each group). Data presented is from a representative experiment. All data are expressed as the mean \pm standard deviation. ${ }^{*} \mathrm{P}<0.05$, ${ }^{* *} \mathrm{P}<0.01$ and ${ }^{* * *} \mathrm{P}<0.001$ vs. LPS group. LPS, lipopolysaccharide; IL, interleukin; MCP, myeloperoxidase; TNF, tumor necrosis factor; ALI, acute lung injury; rHMGB1, recombinant High mobility group box 1; RT-q, reverse transcription-quantitative; NF, nuclear factor; BMMs, bone-marrow derived macrophages; TLR, toll-like receptor; RAGE, receptor for advanced glycation end products; BALF, bronchoalveolar lavage fluid; H\&E, hematoxylin and eosin; RS, Rhodobacter sphaeroides; Mac, macrophage; Neu, neutrophil; Lym, lymphocyte. 
A
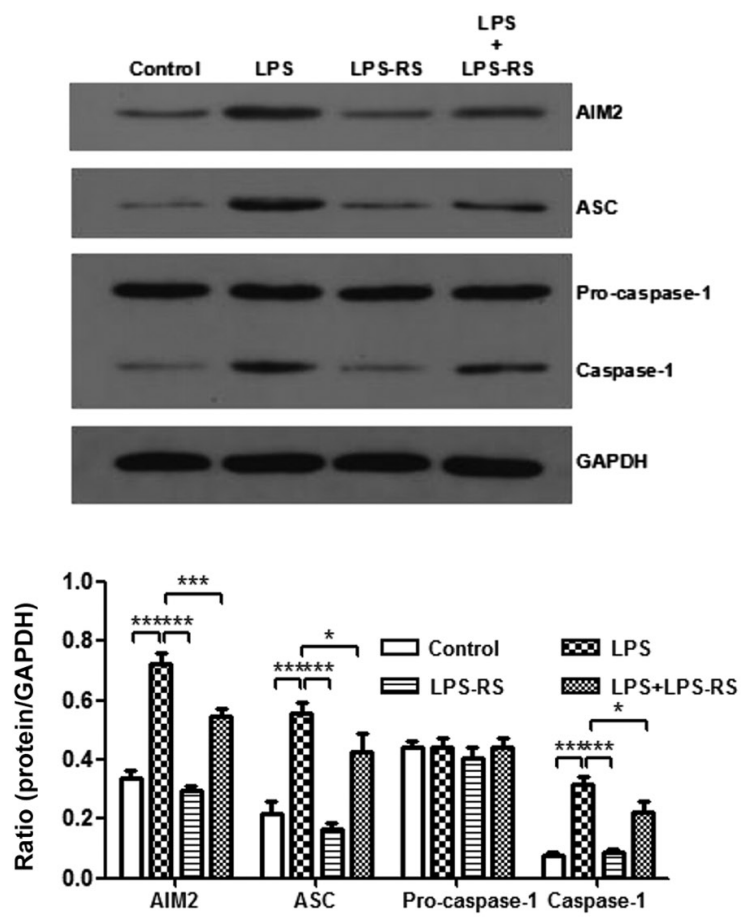

C
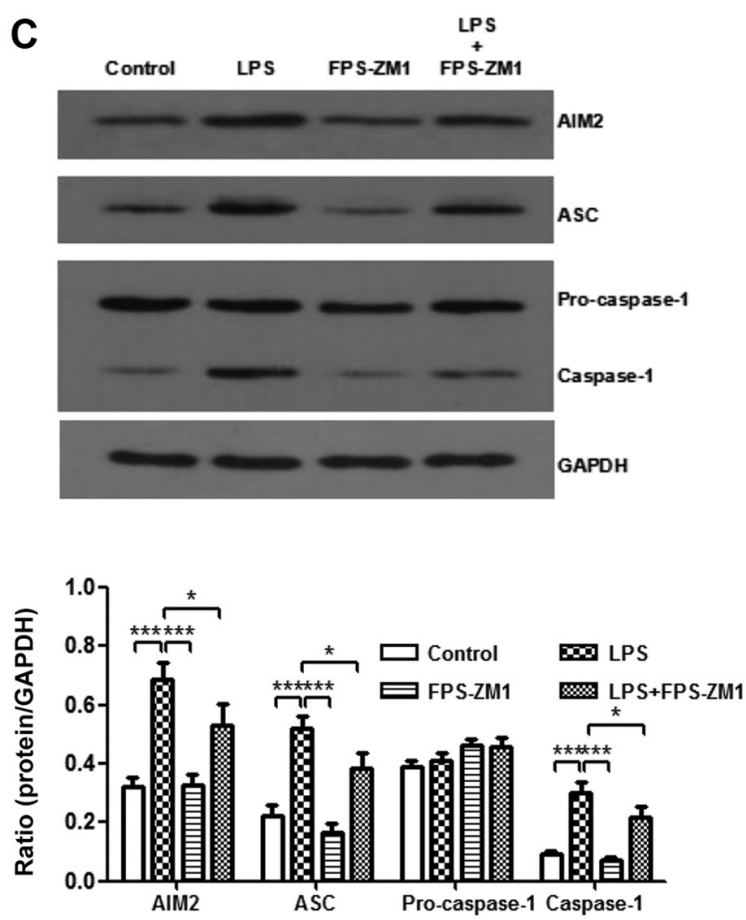

B

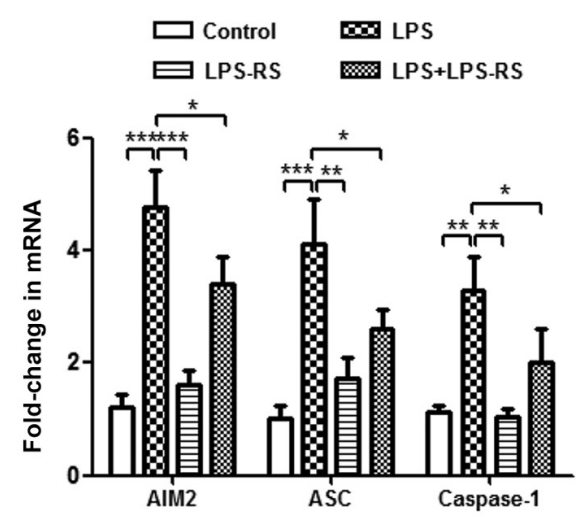

D

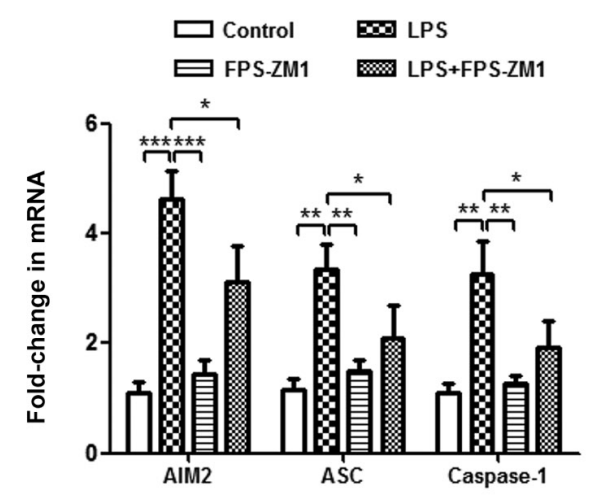

Figure 6. Expression level of the AIM2 inflammasome is downregulated by administration of Toll-like receptor $2 / 4$ or receptor for advanced glycation end products antagonists. In the in vivo experiment, the expression levels of AIM2 inflammasome and GAPDH were detected by western blotting following treatment with (A) LPS-RS and (C) FPS-ZM1. Additionally, RT-qPCR following treatment with (B) LPS-RS and (D) FPS-ZM1 was performed. All experiments were repeated more than three times ( $\mathrm{n}=4-6$ mice per each group). Data presented is from a representative experiment. All data are expressed as the mean \pm standard deviation. ${ }^{*} \mathrm{P}<0.05,{ }^{* *} \mathrm{P}<0.01$ and ${ }^{* * * *} \mathrm{P}<0.001$ vs. LPS group.

Inhibition of TLR2, TLR4 and RAGE reduces the inflammatory response in LPS-induced ALI mouse model. The effects of TLR2/4 antagonist (LPS-RS) and RAGE antagonist (FPS-ZM1) on histopathological changes in LPS-induced ALI were measured by H\&E staining. As depicted in Fig. 5A and B, no histological alteration was observed in lung sections of LPS-RS, FPS-ZM1, and control groups. Treatment with LPS-RS and FPS-ZM1 significantly ameliorated LPS-induced lung injury $(\mathrm{P}<0.001)$. In accordance with these results, treatment with LPS-RS or FPS-ZM1 also inhibited the LPS-induced lung W/D ratio and MPO activity (Fig. 5C-E and G). In BALF, a reduced number of inflammatory cells (total cells, macrophages and neutrophils; Fig. 5F and $\mathrm{H}$ ) and attenuated levels of proinflammatory cytokines (TNF- $\alpha$, IL-6, MCP-1, IL-1 $\beta$, and IL-18; Fig. 5I and J) were noted in ALI mice that received LPS-RS or FPS-ZM1. These data demonstrated that inhibi- 
$\mathbf{E}$
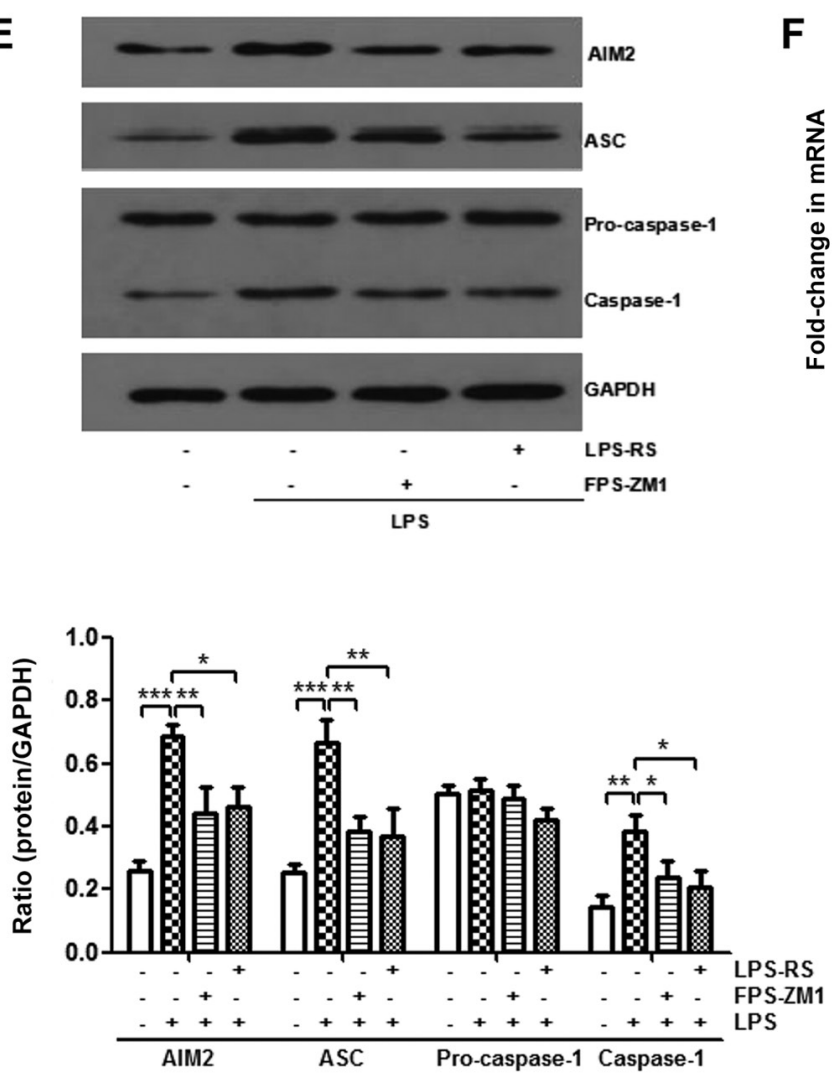

$\mathbf{F}$

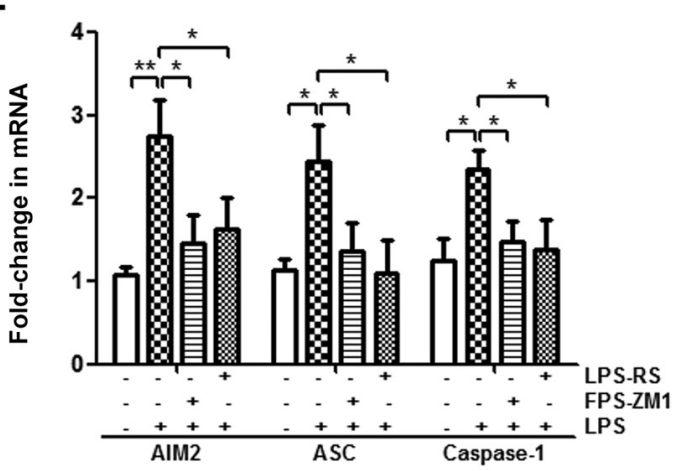

G

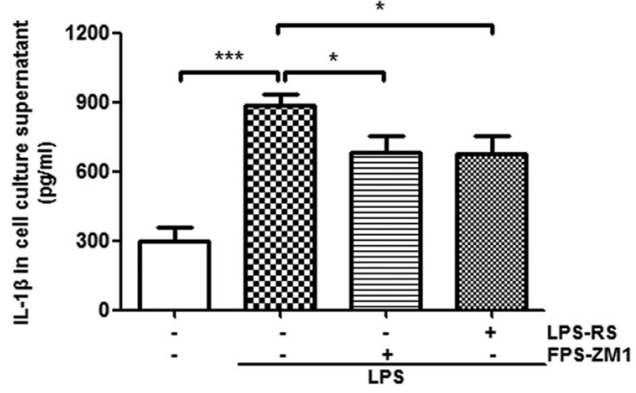

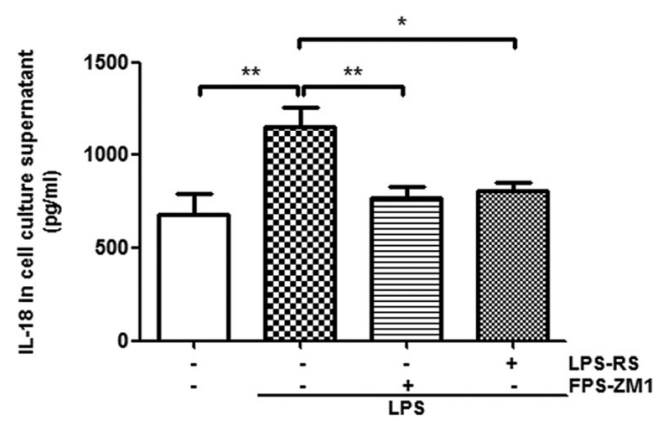

Figure 6. Continued. Expression level of the AIM2 inflammasome is downregulated by administration of Toll-like receptor 2/4 or receptor for advanced glycation end products antagonists. In the in vitro experiment, the expression levels of AIM2 inflammasome and GAPDH in BMMs were also detected by (E) western blotting and (F) RT-qPCR. (G) The expression levels of IL-1 $\beta$ and IL-18 in culture supernatant of BMMs were measured by ELISA. All experiments were repeated more than three times ( $\mathrm{n}=4-6$ mice per each group). Data presented is from a representative experiment. All data are expressed as the mean \pm standard deviation. ${ }^{*} \mathrm{P}<0.05,{ }^{* *} \mathrm{P}<0.01$ and ${ }^{* * *} \mathrm{P}<0.001$ vs. LPS group. LPS, lipopolysaccharide; IL, interleukin; rHMGB1, recombinant High mobility group box 1; RT-q, reverse transcription-quantitative; AIM2, absent in melanoma 2; BMMs, bone-marrow derived macrophages; ASC, apoptosis-associated speck-like protein containing a CARD; RS, Rhodobacter sphaeroides.

tion of TLR2, TLR4 and RAGE reduced the inflammatory response in LPS-induced ALI mouse model.

Inhibition of TLR2, TLR4 and RAGE weakens the activation of the AIM2 inflammasome. It was demonstrated that HMGB1 mediates the activation of the AIM2 inflammasome in macrophages and that HMGB1 acts on macrophages through TLR2, TLR4, and RAGE/NF- $\mathrm{B}$ signaling pathways. Next, whether the AIM2 inflammasome was a downstream mediator of TLR2, TLR4 and RAGE receptors was investigated. LPS-induced increases of AIM2, ASC and caspase-1 protein levels, as well as their mRNA expression levels, were significantly abolished after treatment with LPS-RS or FPS-ZM1 ( $\mathrm{P}<0.05$; Fig. 6A-D). In vitro, compared with those from the LPS-stimulated group, BMMs treated with LPS-RS and FPS-ZM1 significantly decreased mRNA expression levels of AIM2, ASC, and caspase-1 ( $\mathrm{P}<0.05$; Fig. 6E and F). These results suggest that inhibiting TLR2, TLR4 and RAGE weakens the activation of AIM2 inflammasome.

\section{Discussion}

In the present study, using in vivo and in vitro experiments, whether extracellular HMGB1 could activate the AIM2 inflammasome in macrophages, in addition to mediating the polarization of M1 macrophages, to participate in mediating an inflammatory response in ALI through TLR2, TLR4 and $\mathrm{RAGE} / \mathrm{NF}-\kappa \mathrm{B}$ signaling pathways was investigated. An inflam- 
masome can be used as upstream component to promote the secretion of HMGB1 in macrophages and mediate inflammatory reactions. Previous studies demonstrated that extracellular HMGB1 also activated the inflammasome through a number of pathways to further promote the inflammatory response (18-20). Additionally, HMGB1 and the inflammasome have a synergistic influence on each other, causing inflammasome-aggravated tissue damage. However, the relationship between HMGB1 and AIM2 in LPS-induced ALI model has remained elusive. On the other hand, HMGB1 was reported to induce polarization of M1 macrophages in some studies (41-43). Macrophages could secrete and increase the expression level of HMGB1 in the inflammatory reaction. However, the relationship between HMGB1 and polarization M1 macrophages in LPS-induced ALI model needs to be verified.

HMGB1 can activate NF- $\kappa \mathrm{B}$, mediating the inflammatory response after binding to TLR2, TLR4 and RAGE on the surface of inflammatory cells. This classical pathway has been widely recognized and its application in ALI has been confirmed by numerous studies (5-10). However, whether this pathway might be appropriate for the regulation of HMGB1 on macrophages has been confirmed for the first time in the present study to the best of our knowledge. The present results showed that HMGB1 can activate the AIM2 inflammasome in macrophages and promote the polarization of M1 macrophages during the inflammatory reaction of LPS-ALI. The current study confirms that both processes may act through the above-mentioned pathways.

The authors' previous study demonstrated that treatment with anti-HMGB1 had strong anti-inflammatory effects, as shown by decreased infiltration of inflammatory cells, W/D ratio, MPO activity in lung and cytokine production, macrophage and neutrophil infiltration in BALF, as well as strong proinflammatory influences induced by rHMGB1 administration. HMGB1, participating in DNA replication and repair and transcriptional regulation of gene expression, can be passively released by necrotic cells or actively released by macrophages, DCs, and immune cells. HMGB1 is also recognized as a cytokine, owing to its mediation of systemic inflammatory responses through transducing cellular signals (8). HMGB1 has been investigated as a proinflammatory mediator in several acute and chronic immune diseases (44-46). Previous studies demonstrated that HMGB1 plays a pivotal role in monocyte/macrophage and neutrophil activation in the early stage of ALI. HMGB1 not only is an important late mediator of inflammation, but also positively correlates with severity of disease (47). The increase of HMGB1 level in the alveolar spaces might induce neutrophilic influx. HMGB1 is secreted by macrophages and DCs into the alveoli, leading to a severe systemic inflammatory response in ALI $(48,49)$. Administration of rHMGB1 causes pulmonary neutrophil accumulation, in addition to the release of IL-1 $\beta$, TNF- $\alpha$ and MIP-2, enhancing inflammatory responses $(47,50)$. Inhibiting the expression level of HMGB1 may represent an underlying approach for preventing or minimizing ALI (51,52). All those above-mentioned studies are in line with results of the present study.

Additionally, AIM2 is a member of the IFI20X/IFI16 (PYHIN) protein family (53). The AIM2 inflammasome is expressed in macrophages and DCs, and its components are AIM2, ASC, and caspase-1 $(15,16)$. When the AIM2 inflammasomes are activated, ASC, AIM2 and pro-caspase-1 interact with each other, leading to their activation. The activated caspase- 1 cleaves pro-IL- $1 \beta$ and pro-IL-18 into active proinflammatory cytokines. The activation of the AIM2 inflammasome is responsible for processing and secretion of IL-1 $\beta$ and IL-18 (54,55). AIM2 is a major intracellular polyprotein inflammatory pathway for the innate immune system in infection through overproduction of IL-1 $\beta$ and IL-18 (56). Inhibition of the activation of the AIM2 inflammasome is associated with improved ALI and innate immune responses (16,56-58). To further investigate the proinflammatory mechanism of HMGB1 in ALI, in the present research, an attempt was made to study the relationship between HMGB1 and AIM2 inflammasome in macrophages. Research conducted by Liu et al (20) showed that the HMGB1-DNA complex initially induced the activation of the AIM2 inflammasome and also promoted swift release of proinflammatory cytokines (IL-1 $\beta$ ) via RAGE. The effects of HMGB1 on activation of the LPS-induced AIM2 inflammasome in macrophages in in vivo and in vitro experiments were observed. The findings of the current study showed that HMGB1 enhanced the activation of the LPS-induced AIM2 inflammasome in macrophages and promoted the secretion of downstream inflammatory cytokines (IL-1 $\beta$ and IL-18) induced by the AIM2 inflammasome. Therefore, these results suggested that HMGB1 participates in the pathogenesis of ALI through activation of the AIM2 inflammasome.

Macrophages are essential for innate immunity and inflammatory responses, playing a substantial role in lung alveoli and the alveolar space. Inflammation is related to macrophage phenotype and function, and this process has been described during the inflammatory stage of ALI (59). During lung inflammation, macrophages exhibit two main differentiation states, M1 and M2. M1 macrophages produce the proinflammatory cytokines (TNF- $\alpha$, IL-6, IL-1 $\beta$, IL-12 and MCP-1), and inducible nitric oxide synthase in response to interferon- $\gamma(\mathrm{IFN}-\gamma)$. M2 macrophages produce anti-inflammatory cytokines (IL-10 and IL-1RA) in response to IL-4 and IL-13 (34,60,61). M1-polarized macrophages express specific surface markers, including MHC II, CD80, CD86, CD40 and CCR2, whereas M2-polarized macrophages express CD206, dectin-1, transferrin receptor, and CD200R (34). Increasing evidence suggests that polarization of M1 macrophages induces tissue injury, which is involved in the pathogenesis of ALI. In contrast, polarization of M2 macrophages is characterized by reducing the proinflammatory response $(34,62,63)$. The present study observed that the percentage of MHC II, CD80, CD86 and CD40-expressing AMs increased, while the percentage of CD206, IL-10-expressing AMs decreased with administration of rHMGB1. Moreover, opposite changes were found in the anti-HMGB1 group. All the results reflected the HMGB1 induction of polarization of M1 macrophages in LPS-induced ALI. It was also observed that stimulation of rHMGB1 increased the expression levels of M1-related cytokines (TNF- $\alpha$, IL-6 and MCP-1), while decreased the expression levels of M2-related cytokine (IL-10) in culture supernatant of BMMs; however, stimulation of anti-HMGB1 induced the opposite effect, which indirectly proved the above-mentioned conclusion. Taken together, HMGB1 could induce polarization of M1 macrophages in vivo and in vitro. 
HMGB1 has been reported to interact with at least three receptors, RAGE, TLR2 and TLR4, to transmit cellular signals. The RAGE pathway leads to the activation of $\mathrm{NF}-\kappa \mathrm{B}$, as well as promoting secretion of cytokines (TNF- $\alpha$, IL-6 and IFN- $\gamma$ ). Activation of TLR2 and TLR4 results in activation of NF- $\mathrm{BB}$ through MyD88 (8). TLR2, TLR4 and RAGE were expressed at high levels in the lungs by a variety of cells, including macrophages, DCs and activated endothelial and vascular smooth muscle cells $(8,64,65)$. To explore the signaling pathway between HMGB1 and the AIM2 inflammasome in macrophages, it was evaluated whether TLR2, TLR4, and RAGE/NF- $\mathrm{B}$ signaling pathways participated in mediating these two inflammatory mediator effects $(5,25,39,40)$. The results of the present study showed that extracellular HMGB1 could enhance the expression levels of components of TLR2, TLR4 and RAGE/NF- $\kappa$ B signaling pathways in macrophages. Consistent with the present results, several studies showed that HMGB1 stimulated the inflammatory response through RAGE, TLR2 and TLR4, and was associated with the upregulation of RAGE, TLR2, TLR4, and NF- $\kappa$ B expression $(23,25,38)$. In the current research, it was found that HMGB1 upregulated the expression levels of components of TLR2 TLR4 and RAGE/NF- $\kappa$ B signaling pathways. To further assess whether HMGB1 could activate the AIM2 inflammasome in macrophages through TLR2, TLR4 and RAGE/NF- $\mathrm{B}$ signaling pathways, TLR2/4 (LPS-RS) and RAGE antagonists (FPS-ZM1) were used to intervene on expression of AIM2 inflammasome in vivo and in vitro. The present results showed that LPS-RS and FPS-ZM1 reduced LPS-induced inflammatory response in ALI mouse model, weakened activation and expression of LPS-induced AIM2 inflammasome in macrophages, and inhibited AIM2 inflammasome-induced secretion of downstream inflammatory cytokines (IL-1 $\beta$ and IL-18). Therefore, it can be concluded that extracellular HMGB1 activated and upregulated TLR2, TLR4, and RAGE/NF- $\mathrm{BB}$ signaling pathways to further activate the AIM2 inflammasome in macrophages, participating in the pathogenesis of ALI.

The current study illustrates the importance of the mechanism of HMGB1 regulation on macrophages. In the treatment of ALI induced by LPS, to downregulate polarization of M1 macrophages or to promote polarization of M2 macrophages, or to inhibit the expression of AIM2 inflammasome, or to apply anti-HMGB1 to decrease HMGB1, or to apply TLR inhibitor, or RAGE inhibitor to downregulate inflammatory response were used as control targets to successfully treat ALI. As a result, to test the levels of inflammatory factors and decrease their high level through these strategies in ALI patients are the therapeutic goals of this study. Some anti-inflammatory drugs are widely applied in clinical practice though some inhibitors are still being applied in experimental studies and are a certain distance away from clinical application.

However, further studies are required to thoroughly study the complex signaling pathways between extracellular HMGB1 and AIM2 inflammasomes in macrophages in ALI, in addition to via TLR2, TLR4, and RAGE/NF-кB.

In conclusion, HMGB1 participated in the pathological mechanism of LPS-induced ALI by activating the AIM2 inflammasomes in macrophages, as well as inducing polarization of M1 macrophages through TLR2, TLR4 and $\mathrm{RAGE} / \mathrm{NF}-\kappa \mathrm{B}$ signaling pathways.

\section{Acknowledgements}

Not applicable.

\section{Funding}

This study was financially supported by the National Natural Science Foundation of China (grant no. 81571946). The authors would like to thank staff in the Medical Science Research Center of Zhongnan Hospital for their assistance (Wuhan, China).

\section{Availability of data and materials}

The datasets used and/or analyzed during the current study are available from the corresponding author on reasonable request.

\section{Authors' contributions}

JW and RL carried out the studies, participated in data collection, and drafted the manuscript. $\mathrm{BH}$ and $\mathrm{XR}$ performed the statistical analysis and participated in study design. JL and ZP participated in acquisition, analysis, or interpretation of data, and drafted the manuscript as well. All the authors studied and approved the submitted version of manuscript.

\section{Ethics approval and consent to participate}

All animal experiments were approved by the Institutional Animal Care and Use Committee of Wuhan University (Wuhan, China), as well as complying with the Animal Welfare Act.

\section{Patient consent for publication}

Not applicable.

\section{Competing interests}

The authors declare that they have no conflicts of interest.

\section{References}

1. Matthay MA, Ware LB and Zimmerman GA: The acute respiratory distress syndrome. J Clin Invest 122: 2731-2740, 2012.

2. Bellani G, Laffey JG, Pham T, Fan E, Brochard L, Esteban A, Gattinoni L, van Haren F, Larsson A, McAuley DF, et al; LUNG SAFE Investigators; ESICM Trials Group: Epidemiology, Patterns of Care, and Mortality for Patients With Acute Respiratory Distress Syndrome in Intensive Care Units in 50 Countries. JAMA 315: 788-800, 2016.

3. Levy BD and Serhan CN: Resolution of acute inflammation in the lung. Annu Rev Physiol 76: 467-492, 2014.

4. Standiford TJ and Ward PA: Therapeutic targeting of acute lung injury and acute respiratory distress syndrome. Transl Res 167: 183-191, 2016.

5. Kang R, Chen R, Zhang Q, Hou W, Wu S, Cao L, Huang J, Yu Y, Fan XG, Yan Z, et al: HMGB1 in health and disease. Mol Aspects Med 40: 1-116, 2014

6. Bae JS: Role of high mobility group box 1 in inflammatory disease: Focus on sepsis. Arch Pharm Res 35: 1511-1523, 2012.

7. Lotze MT and Tracey KJ: High-mobility group box 1 protein (HMGB1): Nuclear weapon in the immune arsenal. Nat Rev Immunol 5: 331-342, 2005.

8. Klune JR, Dhupar R, Cardinal J, Billiar TR and Tsung A: HMGB1: Endogenous danger signaling. Mol Med 14: 476-484, 2008. 
9. Ueno H, Matsuda T, Hashimoto S, Amaya F, Kitamura Y, Tanaka M, Kobayashi A, Maruyama I, Yamada S, Hasegawa N, et al: Contributions of high mobility group box protein in experimental and clinical acute lung injury. Am J Respir Crit Care Med 170: 1310-1316, 2004.

10. Sundén-Cullberg J, Norrby-Teglund A, Rouhiainen A, Rauvala H, Herman G, Tracey KJ, Lee ML, Andersson J, Tokics L and Treutiger CJ: Persistent elevation of high mobility group box-1 protein (HMGB1) in patients with severe sepsis and septic shock. Crit Care Med 33: 564-573, 2005.

11. Mosser DM and Edwards JP: Exploring the full spectrum of macrophage activation. Nat Rev Immunol 8: 958-969, 2008.

12. Lee JW, Park JW, Shin NR, Park SY, Kwon OK, Park HA, Lim Y, Ryu HW, Yuk HJ, Kim JH, et al: Picrasma quassiodes (D. Don) Benn. attenuates lipopolysaccharide (LPS)-induced acute lung injury. Int J Mol Med 38: 834-844, 2016.

13. Duan JX, Zhou Y, Zhou AY, Guan XX, Liu T, Yang HH, Xie H and Chen P: Calcitonin gene-related peptide exerts anti-inflammatory property through regulating murine macrophages polarization in vitro. Mol Immunol 91: 105-113, 2017

14. Liu YC, Zou XB, Chai YF and Yao YM: Macrophage polarization in inflammatory diseases. Int J Biol Sci 10: 520-529, 2014

15. de Zoete MR, Palm NW, Zhu S and Flavell RA: Inflammasomes. Cold Spring Harb Perspect Biol 6: a016287, 2014.

16. Zhang H, Luo J, Alcorn JF, Chen K, Fan S, Pilewski J, Liu A, Chen W, Kolls JK and Wang J: AIM2 Inflammasome Is Critical for Influenza-Induced Lung Injury and Mortality. J Immunol 198: 4383-4393, 2017

17. Kang R, Chen R, Xie M, Cao L, Lotze MT, Tang D and Zeh HJ III: The Receptor for Advanced Glycation End Products Activates the AIM2 Inflammasome in Acute Pancreatitis. J Immunol 196 4331-4337, 2016

18. Ngoungoure FP and Owona BA: Withaferin A modulates AIM2 inflammasome and caspase-1 expression in THP-1 polarized macrophages. Exp Cell Res 383: 111564, 2019.

19. Vande Walle L, Kanneganti TD and Lamkanfi M: HMGB1 release by inflammasomes. Virulence 2: 162-165, 2011.

20. Liu L, Yang M, Kang R, Dai Y, Yu Y, Gao F, Wang H, Sun X, Li X, Li J, et al: HMGB1-DNA complex-induced autophagy limits AIM2 inflammasome activation through RAGE. Biochem Biophys Res Commun 450: 851-856, 2014.

21. Sun Q, Loughran P, Shapiro R, Shrivastava IH, Antoine DJ, Li T, Yan Z, Fan J, Billiar TR and Scott MJ: Redox-dependent regulation of hepatocyte absent in melanoma 2 inflammasome activation in sterile liver injury in mice. Hepatology 65: 253-268, 2017.

22. Zhou H, Wang Y, Wang W, Jia J, Li Y, Wang Q, Wu Y and Tang J: Generation of monoclonal antibodies against highly conserved antigens. PLoS One 4: e6087, 2009.

23. Han L, Zhang M, Wang M, Jia J, Zhao M, Fan Y and Li X: High Mobility Group Box-1 Promotes Inflammation-Induced Lymphangiogenesis via Toll-Like Receptor 4-Dependent Signalling Pathway. PLoS One 11: e0154187, 2016.

24. Wang C, Liu XX, Huang KB, Yin SB, Wei JJ, Hu YF, Gu Y and Zheng GQ: Preconditioning with recombinant high-mobility group box 1 induces ischemic tolerance in a rat model of focal cerebral ischemia-reperfusion. J Neurochem 137: 576-588, 2016.

25. Chen Y, Huang XJ, Yu N, Xie Y, Zhang K, Wen F, Liu H and Di Q: HMGB1 Contributes to the Expression of P-Glycoprotein in Mouse Epileptic Brain through Toll-Like Receptor 4 and Receptor for Advanced Glycation End Products. PLoS One 10: $\mathrm{e} 0140918,2015$.

26. Abdelmageed ME, El-Awady MS, Abdelrahim $M$ and Suddek GM: LPS-RS attenuation of lipopolysaccharide-induced acute lung injury involves NF- $\kappa \mathrm{B}$ inhibition. Can J Physiol Pharmacol 94: 140-146, 2016.

27. Krikun G, Trezza J, Shaw J, Rahman M, Guller S, Abrahams VM and Lockwood CJ: Lipopolysaccharide appears to activate human endometrial endothelial cells through TLR-4-dependent and TLR-4-independent mechanisms. Am J Reprod Immunol 68: 233-237, 2012

28. Deane R, Singh I, Sagare AP, Bell RD, Ross NT, LaRue B Love R, Perry S, Paquette N, Deane RJ, et al: A multimodal RAGE-specific inhibitor reduces amyloid $\beta$-mediated brain disorder in a mouse model of Alzheimer disease. J Clin Invest 122: 1377-1392, 2012

29. Zhong WT, Wu YC, Xie XX, Zhou X, Wei MM, Soromou LW, Ci XX and Wang DC: Phillyrin attenuates LPS-induced pulmonary inflammation via suppression of MAPK and NF- $\kappa$ B activation in acute lung injury mice. Fitoterapia 90: 132-139, 2013
30. Xiao X, Yang M, Sun D and Sun S: Curcumin protects against sepsis-induced acute lung injury in rats. J Surg Res 176: e31-e39, 2012.

31. Livak KJ and Schmittgen TD: Analysis of relative gene expression data using real-time quantitative PCR and the 2(-Delta Delta C(T)) Method. Methods 25: 402-408, 2001.

32. Zhang F, Huang G, Hu B, Fang LP, Cao EH, Xin XF, Song Y and Shi Y: Anti-HMGB1 neutralizing antibody ameliorates neutrophilic airway inflammation by suppressing dendritic cell-mediated Th17 polarization. Mediators Inflamm 2014: 257930, 2014.

33. Zhang F, Huang G, Hu B, Qian GS and Song Y: Recombinant HMGB1 A box protein inhibits Th17 responses in mice with neutrophilic asthma by suppressing dendritic cell-mediated Th17 polarization. Int Immunopharmacol 24: 110-118, 2015.

34. Aggarwal NR, King LS and D'Alessio FR: Diverse macrophage populations mediate acute lung inflammation and resolution. Am J Physiol Lung Cell Mol Physiol 306: L709-L725, 2014.

35. Nie H, Wang A, He Q, Yang Q, Liu L, Zhang G, Huang Y, Ding X, Yu H and $\mathrm{Hu}$ S: Phenotypic switch in lung interstitial macrophage polarization in an ovalbumin-induced mouse model of asthma. Exp Ther Med 14: 1284-1292, 2017.

36. Mathie SA, Dixon KL, Walker SA, Tyrrell V, Mondhe M, O'Donnell VB, Gregory LG and Lloyd CM: Alveolar macrophages are sentinels of murine pulmonary homeostasis following inhaled antigen challenge. Allergy 70: 80-89, 2015.

37. Pilzweger $\mathrm{C}$ and Holdenrieder S: Circulating HMGB1 and RAGE as Clinical Biomarkers in Malignant and Autoimmune Diseases. Diagnostics (Basel) 5: 219-253, 2015.

38. Fang F and Jiang D: IL-1//HMGB1 signalling promotes the inflammatory cytokines release via TLR signalling in human intervertebral disc cells. Biosci Rep 36: pii: e00379, 2016.

39. Watanabe K, Karuppagounder V, Arumugam S, Thandavarayan RA, Pitchaimani V, Sreedhar R, Afrin R, Harima M, Suzuki H, Suzuki K, et al: Pruni cortex ameliorates skin inflammation possibly through HMGB1-NFאB pathway in house dust mite induced atopic dermatitis $\mathrm{NC} / \mathrm{Nga}$ transgenic mice. J Clin Biochem Nutr 56: 186-194, 2015.

40. Kang N, Hai Y, Yang J, Liang F and Gao CJ: Hyperbaric oxygen intervention reduces secondary spinal cord injury in rats via regulation of HMGB1/TLR4/NF- $\kappa$ B signaling pathway. Int J Clin Exp Pathol 8: 1141-1153, 2015.

41. Tian S, Zhang L, Tang J, Guo X, Dong K and Chen SY: HMGB exacerbates renal tubulointerstitial fibrosis through facilitating M1 macrophage phenotype at the early stage of obstructive injury. Am J Physiol Renal Physiol 308: F69-F75, 2015.

42. Son M, Porat A, He M, Suurmond J, Santiago-Schwarz F, Andersson U, Coleman TR, Volpe BT, Tracey KJ, Al-Abed Y, et al: C1q and HMGB1 reciprocally regulate human macrophage polarization. Blood 128: 2218-2228, 2016

43. Schaper F, de Leeuw K, Horst G, Bootsma H, Limburg PC, Heeringa P, Bijl M and Westra J: High mobility group box 1 skews macrophage polarization and negatively influences phagocytosis of apoptotic cells. Rheumatology (Oxford) 55: 2260-2270, 2016.

44. Cavone L, Cuppari C, Manti S, Grasso L, Arrigo T, Calamai L, SalpietroC and Chiarugi A: Increase in the Level of Proinflammatory Cytokine HMGB1 in Nasal Fluids of Patients With Rhinitis and its Sequestration by Glycyrrhizin Induces Eosinophil Cell Death. Clin Exp Otorhinolaryngol 8: 123-128, 2015

45. Cuppari C, Manti S, Chirico V, Caruso R, Salpietro V, Giacchi V, Laganà F, Arrigo T, Salpietro C and Leonardi S: Sputum high mobility group box-1 in asthmatic children: A noninvasive sensitive biomarker reflecting disease status. Ann Allergy Asthma Immunol 115: 103-107, 2015.

46. Gangemi S, Casciaro M, Trapani G, Quartuccio S, Navarra M, Pioggia G and Imbalzano E: Association between HMGB1 and COPD: A Systematic Review. Mediators Inflamm 2015: 164913, 2015.

47. Kim JY, Park JS, Strassheim D, Douglas I, Diaz del Valle F, Asehnoune K, Mitra S, Kwak SH, Yamada S, Maruyama I, et al: HMGB1 contributes to the development of acute lung injury after hemorrhage. Am J Physiol Lung Cell Mol Physiol 288: L958-L965, 2005.

48. Nogueira-Machado JA and de Oliveira Volpe CM: HMGB-1 as a target for inflammation controlling. Recent Pat Endocr Metab Immune Drug Discov 6: 201-209, 2012.

49. Lamkanfi M, Sarkar A, Vande Walle L, Vitari AC, Amer AO, Wewers MD, Tracey KJ, Kanneganti TD and Dixit VM: Inflammasome-dependent release of the alarmin HMGB1 in endotoxemia. J Immunol 185: 4385-4392, 2010 
50. Deng Y, Yang Z, Gao Y, Xu H, Zheng B, Jiang M, Xu J, He Z and Wang X: Toll-like receptor 4 mediates acute lung injury induced by high mobility group box-1. PLoS One 8: e64375, 2013.

51. Hidaka S, Iwasaka H, Hagiwara $\mathrm{S}$ and Noguchi T: Gabexate mesilate inhibits the expression of HMGB1 in lipopolysaccharide-induced acute lung injury. J Surg Res 165: 142-150, 2011

52. Entezari M, Javdan M, Antoine DJ, Morrow DM, Sitapara RA, Patel V, Wang M, Sharma L, Gorasiya S, Zur M, et al: Inhibition of extracellular HMGB1 attenuates hyperoxia-induced inflammatory acute lung injury. Redox Biol 2: 314-322, 2014.

53. Ludlow LE, Johnstone RW and Clarke CJ: The HIN-200 family: More than interferon-inducible genes? Exp Cell Res 308: 1-17, 2005.

54. Rathinam VA, Jiang Z, Waggoner SN, Sharma S, Cole LE, Waggoner L, Vanaja SK, Monks BG, Ganesan S, Latz E, et al: The AIM2 inflammasome is essential for host defense against cytosolic bacteria and DNA viruses. Nat Immunol 11: 395-402, 2010.

55. Hu S, Peng L, Kwak YT, Tekippe EM, Pasare C, Malter JS, Hooper LV and Zaki MH: The DNA Sensor AIM2 Maintains Intestinal Homeostasis via Regulation of Epithelial Antimicrobial Host Defense. Cell Rep 13: 1922-1936, 2015.

56. Fernandes-Alnemri T, Yu JW, Juliana C, Solorzano L, Kang S, Wu J, Datta P, McCormick M, Huang L, McDermott E, et al The AIM2 inflammasome is critical for innate immunity to Francisella tularensis. Nat Immunol 11: 385-393, 2010.

57. Man SM, Karki R and Kanneganti TD: AIM2 inflammasome in infection, cancer, and autoimmunity: Role in DNA sensing, inflammation, and innate immunity. Eur J Immunol 46: 269-280, 2016.

58. Karki R, Man SM, Malireddi RKS, Gurung P, Vogel P,Lamkanfi M and Kanneganti TD: Concerted activation of the AIM2 and NLRP3 inflammasomes orchestrates host protection against Aspergillus infection. Cell Host Microbe 17: 357-368, 2015.
59. Herold S, Mayer K and Lohmeyer J: Acute lung injury: How macrophages orchestrate resolution of inflammation and tissue repair. Front Immunol 2: 65, 2011.

60. Lawrence $\mathrm{T}$ and Natoli G: Transcriptional regulation of macrophage polarization: Enabling diversity with identity. Nat Rev Immunol 11: 750-761, 2011.

61. Gordon S and Martinez FO: Alternative activation of macrophages: Mechanism and functions. Immunity 32: 593-604, 2010.

62. Davis MJ, Tsang TM, Qiu Y, Dayrit JK, Freij JB, Huffnagle GB and Olszewski MA: Macrophage M1/M2 polarization dynamically adapts to changes in cytokine microenvironments in Cryptococcus neoformans infection. MBio 4: e00264-e13, 2013.

63. Lu G, Zhang R, Geng S, Peng L, Jayaraman P, Chen C, Xu F, Yang J, Li Q, Zheng H, et al: Myeloid cell-derived inducible nitric oxide synthase suppresses M1 macrophage polarization. Nat Commun 6: 6676, 2015.

64. Wolf L, Herr C, Niederstraßer J, Beisswenger C and Bals R: Receptor for advanced glycation endproducts (RAGE) maintains pulmonary structure and regulates the response to cigarette smoke. PLoS One 12: e0180092, 2017.

65. Fallah MP, Chelvarajan RL, Garvy BA and Bondada S: Role of phosphoinositide 3-kinase-Akt signaling pathway in the age-related cytokine dysregulation in splenic macrophages stimulated via TLR-2 or TLR-4 receptors. Mech Ageing Dev 132: 274-286, 2011.

This work is licensed under a Creative Commons Attribution-NonCommercial-NoDerivatives 4.0 International (CC BY-NC-ND 4.0) License. 